\title{
A novel time efficient structure-preserving splitting method for the solution of two-dimensional reaction-diffusion systems
}

\author{
Nauman Ahmed ${ }^{1,8^{*}}$ (D), Alper Korkmaz ${ }^{2}$, M. Rafiq ${ }^{3}$, Dumitru Baleanu ${ }^{4,5,6}$, Ali Saleh Alshomrani ${ }^{7}$, \\ M.A. Rehman ${ }^{1}$ and M.S. $|q b a|^{8}$
}

Correspondence:

nauman.ahmd01@gmail.com

1 Department of Mathematics,

University of Management and

Technology, Lahore, Pakistan

${ }^{8}$ Department Mathematics and

Statistics, The University of Lahore,

Lahore, Pakistan

Full list of author information is

available at the end of the article

\section{勿 Springer}

\begin{abstract}
In this article, the first part is concerned with the important questions related to the existence and uniqueness of solutions for nonlinear reaction-diffusion systems. Secondly, an efficient positivity-preserving operator splitting nonstandard finite difference scheme (NSFD) is designed for such a class of systems. The presented formulation is unconditionally stable as well as implicit in nature and even time efficient. The proposed NSFD operator splitting technique also preserves all the important properties possessed by continuous systems like positivity, convergence to the fixed points of the system, and boundedness. The proposed algorithm is implicit in nature but more efficient in time than the extensively used Euler method.
\end{abstract}

Keywords: Operator splitting finite difference scheme; Reaction-diffusion models; Positivity; Numerical simulations

\section{Introduction}

Reaction-diffusion equations generally arise in different chemical and biological models which describe the physical phenomena like concentration, density, population sizes, and many more. The negative solution of these systems is meaningless because the solutions describe the concentration, density, and population sizes, and similar type of quantities cannot be negative. Therefore the behavior of the numerical method used to solve such systems must be the same as the behavior of continuous systems. The NSFD method proposed by Mickens [1] is an efficient way to design structure-preserving finite difference (FD) schemes. Various writers proposed NSFD and positivity preserving FD schemes for the solution of differential equations. Many authors used the NSFD technique to find the solution of ordinary differential equations arising in chemical and biological models, for the readers, some of the references are presented [2-8]. In a similar way, different NSFD and positivity preserving FD methods are introduced in the literature to solve reactiondiffusion equations [9-17]. Also, various techniques are used to solve a similar type of diffusion systems [18-23].

(c) The Author(s) 2020. This article is licensed under a Creative Commons Attribution 4.0 International License, which permits use, sharing, adaptation, distribution and reproduction in any medium or format, as long as you give appropriate credit to the original author(s) and the source, provide a link to the Creative Commons licence, and indicate if changes were made. The images or other third party material in this article are included in the article's Creative Commons licence, unless indicated otherwise in a credit line to the material. If material is not included in the article's Creative Commons licence and your intended use is not permitted by statutory regulation or exceeds the permitted use, you will need to obtain permission directly from the copyright holder. To view a copy of this licence, visit http://creativecommons.org/licenses/by/4.0/. 
Many numerical methods have gained considerable attention, as exact solution of many chemical and biological models involving partial differential equations is an uphill task. Therefore many authors proposed different numerical techniques to find the numerical solution of ordinary, partial, and fractional differential equations arising in the models of chemistry, physics, biology, engineering, and many other fields of different sciences [24-36]. The proposed NSFD method introduced in this work is concerned with time efficiency as well as preserving of the properties possessed by the continuous reactiondiffusion models. The proposed method is an operator splitting NSFD method. The operator splitting FD technique introduced by Yanenko and his collaborators is a remarkable achievement for the numerical solution to the problems in theoretical mechanics [37]. An explicit forward Euler FD method is compared with the proposed method. The proposed operator splitting NSFD method maintains the same numerical accuracy and reduces the computation time efficiently. Reflecting numerically, more accuracy requires less CPU time; furthermore, easy implementation enhances the applicability of the proposed NSFD scheme.

The major motivation for the current research is to construct the solutions under special physical traits that lead to the computational difficulties. The developed algorithm not only preserves the necessary properties of the solution, but also reduces computational cost. Furthermore, the existence of solutions of the underlying problem is assured.

\section{Existence of solution}

The goal of the paper is to solve the following system in a square domain:

$$
\partial_{t} w_{i}=d_{w_{i}} \Delta_{2} w_{i}+f\left(t, x, y, w_{i}\right), \quad t>0, a<x, y<b=\Omega, i=1,2,
$$

subject to the initial conditions

$$
w_{i}(x, y, 0)=g_{i}(x, y), \quad \bar{\Omega}=a \leq x, y \leq b
$$

and the homogeneous Neumann boundary conditions

$$
\frac{\partial w_{i}}{\partial \mathbf{n}_{\mathbf{i}}}=0 \quad \text { on } \partial \Omega, i=1,2 \text {, }
$$

where $d_{w_{i}}$ are the diffusion coefficients and are constants, while $g_{i}(x, y)$ are continuous in $\bar{\Omega}$. The conventional integration leads to the inversion of the partial differential operator $\partial_{t}$ by simple integration in the following new analytic form:

$$
w_{i}(x, y, t)=g_{i}(x, y)+\int_{0}^{t} \mathcal{F}\left(\Delta_{2} w_{i}(s)+f\left(x, y, s, w_{i}(s)\right)\right) d s .
$$

In the operator form we can write

$$
T_{i}=T w_{i}(x, y, t)=g_{i}(x, y)+\int_{0}^{t} \mathcal{F}(s) d s,
$$

where

$$
\mathcal{F}=\mathcal{F}\left(\Delta_{2} w_{i}+f\left(x, y, t, w_{i}\right)\right) .
$$


Clearly the solutions $w_{i}$ of system (1) are twice continuously differentiable in the domain $\Omega$ and continuously differentiable in the time domain $[0, T], T>0$. Hence $\mathcal{F}$ in (6) is continuous and bounded, i.e., $|\mathcal{F}|<M, M$ is a positive real number. For the existence of the solution of system (1)-(2),

$$
\begin{aligned}
& T_{i}=g_{i}(x, y)+\int_{0}^{t} \mathcal{F}(s) d s, \\
& \left\|T_{i}\right\|=\left|g_{i}(x, y)\right|+\int_{0}^{t}\|\mathcal{F}\| d s \\
& \quad \leq \kappa_{i}+M . \rho .
\end{aligned}
$$

Hence $T_{i}$ are bounded, and further if we consider a closed and convex subset $B_{r}(\Theta)$ (centered at zero element) in the space of continuous functions with radius $r$, thus

$$
\rho \leq \frac{r-\kappa_{i}}{M}
$$

which gives restriction on the bounds $\kappa_{i}$ of the initial values.

Now consider the family of images $T_{i}^{j}$ of pre-images $w_{i}^{j}$, then from (5) we get the following difference:

$$
\begin{aligned}
T_{i}^{j}(x, y, t)-T_{i}^{j}\left(x, y, t^{*}\right) & =\int_{0}^{t} \mathcal{F}(s) d s-\int_{0}^{t^{*}} \mathcal{F}(s) d s \\
& =\int_{t^{*}}^{t} \mathcal{F}(s) d s, \\
\left\|T_{i}^{j}(x, y, t)-T_{i}^{j}\left(x, y, t^{*}\right)\right\| & \leq M \cdot\left|t-t^{*}\right| .
\end{aligned}
$$

This implies that $T_{i}^{j}$ is equicontinuous, so by the Arzela-Ascoli theorem there exists a subsequence $T_{i}^{j l}$ of $T_{i}^{j}$ which is uniformly convergent, and hence the operator $T$ in (5) turns out to be relatively compact, so the Schauder fixed point theorem is applicable. Summing up the above arguments, the following theorems have been proved.

Theorem 1 Suppose that the functions $w_{i}(x, y, t)$ are twice continuously differentiable in $\Omega=a<x, y<b$, continuously differentiable with respect to the time domain $[0, T]$ along with continuous initial values, then problem (1)-(2) is equivalent to the fixed point operator (5).

Theorem 2 Let $w_{i}(x, y, t)$ be $C^{2}(\Omega)$ and $C^{1}[0, T]$, then the initial boundary value problem for (1) and (2) is solvable for a Schauder fixed point theorem and consequently possesses the solution.

Suppose the right-hand side of $\mathcal{F}\left(w_{i}\right)$ of (1) satisfies the following condition:

$$
\begin{aligned}
\mathcal{F}\left(w_{i}^{1}\right)-\mathcal{F}\left(w_{i}^{2}\right) & =w_{i_{x x}}^{1}+w_{i_{y y}}^{1}-w_{i_{x x}}^{2}-w_{i_{y y}}^{2}+f\left(w_{i}^{1}\right)-f\left(w_{i}^{2}\right) \\
& =w_{i_{x x}}^{1}-w_{i_{x x}}^{2}+w_{i_{y y}}^{1}-w_{i_{y y}}^{2}+f\left(w_{i}^{1}\right)-f\left(w_{i}^{2}\right) .
\end{aligned}
$$


Suppose that $f$ is Lipschitz continuous in $w_{i}$,

$$
\begin{aligned}
\left\|\mathcal{F}\left(w_{i}^{1}\right)-\mathcal{F}\left(w_{i}^{2}\right)\right\| & \leq \mathcal{L}_{1}\left\|w_{i}^{1}-w_{i}^{2}\right\|_{C^{2}(\Omega)}+\mathcal{L}_{2}\left\|w_{i}^{1}-w_{i}^{2}\right\|_{C^{2}(\Omega)}+\mathcal{L}_{3}\left\|w_{i}^{1}-w_{i}^{2}\right\|_{C^{0}(\Omega)} \\
& \leq \mathcal{L}_{1}\left\|w_{i}^{1}-w_{i}^{2}\right\|_{C^{2}(\Omega)}+\mathcal{L}_{2}\left\|w_{i}^{1}-w_{i}^{2}\right\|_{C^{2}(\Omega)}+\mathcal{L}_{3}\left\|w_{i}^{1}-w_{i}^{2}\right\|_{C^{2}(\Omega)}, \\
\left\|\mathcal{F}\left(w_{i}^{1}\right)-\mathcal{F}\left(w_{i}^{2}\right)\right\| & \leq \mathcal{L}\left\|w_{i}^{1}-w_{i}^{2}\right\|_{C^{2}(\Omega)},
\end{aligned}
$$

where

$$
\mathcal{L}=\max \left(\mathcal{L}_{1}, \mathcal{L}_{2}, \mathcal{L}_{3}\right)
$$

which is a necessary condition to show the unique existence under condition (7). Further,

$$
\begin{aligned}
T^{1}-T^{2}= & \int_{0}^{t}\left(\mathcal{F}\left(w_{i}^{1}(s)\right)-\mathcal{F}\left(w_{i}^{2}(s)\right)\right) d s, \\
\left\|T^{1}-T^{2}\right\| & \leq \mathcal{L} d_{i} \int_{0}^{t} d s \cdot\left\|w_{i}^{1}-w_{i}^{2}\right\|_{C^{2}(\Omega)} \\
& =\mathcal{L} \rho d_{i} \int_{0}^{t} d s \cdot\left\|w_{i}^{1}-w_{i}^{2}\right\|_{C^{2}(\Omega)} .
\end{aligned}
$$

The operator $T$ will be a contraction provided

$$
\rho<\frac{1}{d_{i} \mathcal{L}} .
$$

Finally, we establish the following important theorem for the unique existence of the solution of (1)-(2), i.e., we have the following.

Theorem 3 Suppose that the right-hand side of (1) satisfies the Lipschitz condition of the form (8), then problem (1)-(2) is uniquely solvable provided conditions (7) and (9) are satisfied.

\section{Numerical methods}

Firstly, divide $[a, b]^{2} \times[0, T]$ into $M^{2} \times N$ with space step size $h=(b-a) / M$ and time step size $\tau=T / N$. Grid points are

$$
\begin{aligned}
& x_{l}=l h, \quad l=0,1,2, \ldots, M, \\
& y_{m}=m h, \quad m=0,1,2, \ldots, M, \\
& t_{n}=n \tau, \quad n=0,1,2, \ldots, N .
\end{aligned}
$$

The notation $w_{i l, m}^{n}$ is used for the finite difference approximations of $w_{i}(l h, m h, n \tau)$. We present two different FD techniques, the forward Euler FD technique and the operator splitting NSFD technique. We propose the positivity preserving NSFD operator splitting technique for the solution of two-dimensional reaction-diffusion systems. In order to verify all the attributes of the proposed NSFD technique, we also use the forward Euler FD technique for the purpose of comparison. The forward Euler FD technique is explicit in 
nature, time efficient, and easy to implement. But the forward Euler FD technique has the conditional stability. The proposed operator splitting NSFD scheme is implicit in nature and has unconditional stability. The splitting techniques are very efficient numerical techniques for the solution of differential equations. The splitting techniques are widely used numerical techniques for the solution of different nonlinear physical problems [38-43].

\subsection{Forward Euler FD method}

In the forward Euler FD method, time derivative is approximated by the forward difference, and space derivatives are approximated by central differences. The forward Euler FD scheme for system (1) is given as follows:

$$
\delta_{t} w_{i_{l, m}}^{n}=\delta_{x}^{2} w_{i_{l, m}}^{n}+\delta_{y}^{2} w_{i_{l, m}}^{n}+f\left(w_{i_{l, m}}^{n}\right)
$$

where

$$
\begin{aligned}
& \delta_{t} w_{i_{l, m}}^{n}=\frac{w_{i_{l, m}}^{n+1}-w_{i_{l, m}}^{n}}{\tau}, \\
& \delta_{x}^{2} w_{i_{l, m}}^{n}=\frac{w_{i_{l-1, m}}^{n}-2 w_{i_{l, m}}^{n}+w_{i_{l+1, m}}^{n}}{h^{2}}, \\
& \delta_{y}^{2} w_{i_{l, m}}^{n}=\frac{w_{i_{l, m-1}}^{n}-2 w_{i_{l, m}}^{n}+w_{i_{l, m+1}}^{n}}{h^{2}} .
\end{aligned}
$$

The stability region of the forward Euler FD scheme after linearizing the nonlinear reaction-diffusion is approximately $d_{w_{i}}\left(\tau / h^{2}\right) \leq 1 / 4$.

\subsection{Operator splitting FD method}

In this method, we split system (1) into three steps as follows:

$$
\begin{aligned}
& \frac{1}{3} w_{i_{t}}=f\left(w_{i}\right), \\
& \frac{1}{3} w_{i_{t}}=d_{w_{i}} w_{i_{x x}}, \\
& \frac{1}{3} w_{i_{t}}=d_{w_{i}} w_{i_{y y}}
\end{aligned}
$$

and apply the finite difference approximations on the above equations:

$$
\begin{aligned}
& \frac{w_{i_{l, m}}^{n+\frac{1}{3}}-w_{i_{l, m}}^{n}}{\tau}=f\left(w_{i_{l, m}}^{n}\right), \\
& \frac{w_{i_{l, m}}^{n+\frac{2}{3}}-w_{i_{l, m}}^{n}}{\tau}=\frac{w_{i_{l-1, m}}^{n+\frac{2}{3}}-2 w_{i_{l, m}}^{n+\frac{2}{3}}+w_{i_{l+1, m}}^{n+\frac{2}{3}}}{h^{2}}, \\
& \frac{w_{i_{l, m}}^{n+1}-w_{i_{l, m}}^{n}}{\tau}=\frac{w_{i_{l, m-1}}^{n+1}-2 w_{i_{l, m}}^{n+1}+w_{i_{l, m+1}}^{n+1}}{h^{2}},
\end{aligned}
$$

where $(n+1 / 3)$ means the time step $t_{n+1 / 3}=t_{n}+(1 / 3) \tau,(n+2 / 3)$ means the time step $t_{n+2 / 3}=t_{n}+(2 / 3) \tau$, and $(n+1)$ means the time step $t_{n+1}=t_{n}+\tau$. 


\subsection{NSFD method}

The NSFD schemes give numerical approximations to differential equations by constructing discrete models. These schemes preserve the important physical properties of the continuous model and consequently give reliable results. The following are the rules defined by Mickens in [1] for designing the NSFD scheme:

Rule 1. The discrete derivative should be of the same order as the order of continuous derivative appearing in the model.

Rule 2. The step sizes $h$ and $\tau$ can be replaced with the functions $\Phi(h)$ and $\Psi(\tau)$ with the conditions that if $h \rightarrow 0, \tau \rightarrow 0$ then $\Phi(h) \rightarrow 0, \Psi(\tau) \rightarrow 0$, respectively. Rule 3. Nonlinear terms should, in general, be replaced with nonlocal discrete representations. For example, $w_{1}^{n}{ }_{l, m}^{n} w_{2 l, m}^{n}$ is replaced with $w_{1, m}^{n} w_{2, m}^{n+1}$.

Rule 4 . Any property $P$ demonstrated by the continuous system should be preserved by the numerical method.

After applying these rules, equations (11)-(13) become an NSFD operator splitting scheme.

\subsection{Stability and accuracy of the proposed NSFD method}

The stability and consistency of operator splitting schemes depend on the split solutions $[38,44]$. The time derivative has $O(\tau)$ accuracy, and the reaction step is exactly solved so it is unconditionally stable. In the similar way, the space derivative has $O\left(h^{2}\right)$ accuracy, and the diffusion step is also unconditionally stable.

In the next sections we consider two different chemical reaction models for the application of the positivity-preserving NSFD operator splitting method and the forward Euler method.

\section{Brusselator reaction-diffusion model}

The Brusselator model is an auto-catalytic chemical reaction developed by Prigogine [45]. This Brusselator reaction-diffusion system plays a significant role in the application of cooperative processes of chemical kinetics. This system arises in a large number of physical problems. The Brusselator reaction-diffusion system appears in the formation of ozone by atomic oxygen through a triple collision as well as in enzymatic reactions, and in plasma and laser physics. The system of two-dimensional Brusselator model is

$$
\begin{aligned}
& w_{1_{t}}=d_{w_{1}}\left(w_{1_{x x}}+w_{1_{y y}}\right)+D-(C+1) w_{1}+w_{1}^{2} w_{2}, \\
& w_{2_{t}}=d_{w_{2}}\left(w_{2_{x x}}+w_{2_{y y}}\right)+C w_{1}-w_{1}^{2} w_{2}
\end{aligned}
$$

with the initial condition

$$
\begin{array}{ll}
w_{1}(x, y, 0)=g_{1}(x, y) \geq 0, & a \leq x, y \leq b, \\
w_{2}(x, y, 0)=g_{2}(x, y) \geq 0, & a \leq x, y \leq b
\end{array}
$$

and the homogeneous Neumann boundary conditions. Here, $w_{1}=w_{1}(x, y, t)$ and $w_{2}=$ $w_{2}(x, y, t)$ are the concentrations of the chemical substances. $C$ and $D$ are constant concentrations. The equilibrium point of system $(14)-(15)$ is $\left(w_{1}^{*}, w_{2}^{*}\right)=(D, C / D)$. Twizell et 
al. [49] concluded that system (14)-(15) has a stable equilibrium point $\left(w_{1}^{*}, w_{2}^{*}\right)$ under the condition $1-C+D^{2} \geq 0$ and unstable if $1-C+D^{2}<0$. Now we apply the forward Euler FD scheme (10) on equations (14)-(15), we have

$$
\begin{aligned}
w_{1_{l, m}}^{n+1}= & w_{1_{l, m}}^{n}+\eta_{1}\left(w_{1_{l-1, m}^{n}}^{n}-2 w_{1_{l, m}^{n}}^{n}+w_{1_{l+1, m}}^{n}\right) \\
& +\eta_{1}\left(w_{1_{l, m-1}^{n}}^{n}-2 w_{1_{l, m}}^{n}+w_{1_{l, m+1}}^{n}\right)+\tau D-\tau(C+1) w_{1_{l, m}}^{n} \\
& +\tau\left(w_{1_{l, m}}^{n}\right)^{2} w_{2_{l, m}^{n}}^{n}, \\
w_{2_{l, m}}^{n+1}= & w_{2_{l, m}^{n}}^{n}+\eta_{2}\left(w_{2_{l-1, m}^{n}}^{n}-2 w_{2_{l, m}^{n}}^{n}+w_{2_{l+1, m}}^{n}\right) \\
& +\eta_{2}\left(w_{2_{l, m-1}^{n}}^{n}-2 w_{2_{l, m}}^{n}+w_{2_{l, m+1}^{n}}^{n}\right)+\tau C w_{1_{l, m}}^{n} \\
& -\tau\left(w_{1_{l, m}^{n}}^{n}\right)^{2} w_{2_{l, m}^{n}}^{n}, \\
\eta_{1}=d_{w_{1}} & \frac{\tau}{h^{2}} \quad \text { and } \quad \eta_{2}=d_{w_{2}} \frac{\tau}{h^{2}} .
\end{aligned}
$$

Now we apply the NSFD operator splitting technique (11)-(13) on equation (14). At the first time step, the reaction term is solved:

$$
\frac{w_{1_{l, m}}^{n+\frac{1}{3}}-w_{1_{l, m}}^{n}}{\tau}=D-(C+1) w_{1_{l, m}}^{n+\frac{1}{3}}+\left(w_{1_{l, m}}^{n}\right)^{2} w_{2_{l, m}^{n}}^{n} .
$$

After simplification, we have

$$
w_{1_{l, m}}^{n+\frac{1}{3}}=\frac{w_{1_{l, m}^{n}}^{n}+\tau D+\tau\left(w_{1_{l, m}^{n}}^{n}\right)^{2} w_{2_{l, m}^{n}}^{n}}{1+\tau(C+1)} .
$$

At the second time step, the space derivative w.r.t. $x$ is approximated

$$
\begin{aligned}
& \frac{w_{1_{l, m}}^{n+\frac{2}{3}}-w_{1_{l, m}}^{n}}{\tau}=\frac{w_{1_{l-1, m}}^{n+\frac{2}{3}}-2 w_{1_{l, m}}^{n+\frac{2}{3}}+w_{1_{l+1, m}}^{n+\frac{2}{3}}}{h^{2}}, \\
& -\eta_{1} w_{1_{l-1, m}}^{n+\frac{2}{3}}+\left(1+2 \eta_{1}\right) w_{1_{l, m}}^{n+\frac{2}{3}}-\eta_{1} w_{1_{l+1, m}}^{n+\frac{2}{3}}=w_{1_{l, m}}^{n+\frac{1}{3}} .
\end{aligned}
$$

Similarly, at the third time step the space derivative w.r.t. $y$ is approximated as follows:

$$
-\eta_{1} w_{1_{l, m-1}^{n+1}}^{n+1}+\left(1+2 \eta_{1}\right) w_{1_{l, m}^{n+1}}^{n+}-\eta_{1} w_{1_{l, m+1}^{n+1}}^{n+1}=w_{1_{l, m}}^{n+\frac{2}{3}}
$$

In a similar way, the NSFD operator splitting scheme for equation (15) at all steps is

$$
\begin{aligned}
& w_{2_{l, m}}^{n+\frac{1}{3}}=\frac{w_{2_{l, m}}^{n}+\tau C w_{1_{l, m}}^{n}}{1+\tau\left(w_{1_{l, m}}^{n}\right)^{2}}, \\
& -\eta_{2} w_{2_{l-1, m}}^{n+\frac{2}{3}}+\left(1+2 \eta_{2}\right) w_{2_{l, m}}^{n+\frac{2}{3}}-\eta_{2} w_{2_{l+1, m}}^{n+\frac{2}{3}}=w_{2_{l, m}}^{n+\frac{1}{3}}, \\
& -\eta_{2} w_{2_{l, m-1}^{n+1}}^{n+1}+\left(1+2 \eta_{2}\right) w_{2_{l, m}^{n+1}}^{n+}-\eta_{2} w_{2_{l, m+1}^{n+1}}^{n+1}=w_{2_{l, m}}^{n+\frac{2}{3}} .
\end{aligned}
$$




\subsection{Matrix representation}

Let $\mathbf{w}_{\mathbf{1}}^{\mathbf{n}+\frac{2}{3}}=\left(w_{1_{0, m}}^{n+\frac{2}{3}}, \ldots, w_{1_{M, m}^{n+\frac{2}{3}}}^{n}\right)^{t}$ and $\mathbf{w}_{\mathbf{2}}^{\mathbf{n}+\frac{2}{3}}=\left(w_{2_{0, m}}^{n+\frac{2}{3}}, \ldots, w_{2_{M, m}}^{n+\frac{2}{3}}\right)^{t}$, where $(\cdot)^{t}$ represents the transposition of a vector. Along with the homogeneous Neumann boundary conditions, equations (21) and (24) are equivalent to the matrix form

$$
\begin{aligned}
& B_{1} \mathbf{w}_{\mathbf{1}}^{\mathbf{n}+\frac{2}{3}}=\mathbf{w}_{\mathbf{1}}^{\mathbf{n}+\frac{1}{3}}, \\
& B_{3} \mathbf{w}_{\mathbf{2}}^{\mathbf{n}+\frac{2}{3}}=\mathbf{w}_{\mathbf{2}}^{\mathbf{n}+\frac{1}{3}},
\end{aligned}
$$

where $B_{1}$ and $B_{3}$ are real matrices of size $(M+1) \times(M+1)$

$$
\begin{aligned}
& B_{1}=\left(\begin{array}{cccccccc}
1+2 \eta_{1} & -2 \eta_{1} & 0 & \cdots & \cdots & \cdots & \cdots & 0 \\
-\eta_{1} & 1+2 \eta_{1} & -\eta_{1} & \ddots & & & & \vdots \\
0 & -\eta_{1} & 1+2 \eta_{1} & -\eta_{1} & \ddots & & & \vdots \\
\vdots & \ddots & \ddots & \ddots & \ddots & \ddots & & \vdots \\
\vdots & & \ddots & \ddots & \ddots & \ddots & \ddots & \vdots \\
\vdots & & & \ddots & -\eta_{1} & 1+2 \eta_{1} & -\eta_{1} & 0 \\
\vdots & & & & \ddots & -\eta_{1} & 1+2 \eta_{1} & -\eta_{1} \\
0 & \ldots & \ldots & \ldots & \cdots & 0 & -2 \eta_{1} & 1+2 \eta_{1}
\end{array}\right) \text {, } \\
& B_{3}=\left(\begin{array}{cccccccc}
1+2 \eta_{2} & -2 \eta_{2} & 0 & \cdots & \cdots & \cdots & \cdots & 0 \\
-\eta_{2} & 1+2 \eta_{2} & -\eta_{2} & \ddots & & & & \vdots \\
0 & -\eta_{2} & 1+2 \eta_{2} & -\eta_{2} & \ddots & & & \vdots \\
\vdots & \ddots & \ddots & \ddots & \ddots & \ddots & & \vdots \\
\vdots & & \ddots & \ddots & \ddots & \ddots & \ddots & \vdots \\
\vdots & & & \ddots & -\eta_{2} & 1+2 \eta_{2} & -\eta_{2} & 0 \\
\vdots & & & & \ddots & -\eta_{2} & 1+2 \eta_{2} & -\eta_{2} \\
0 & \ldots & \ldots & \ldots & \ldots & 0 & -2 \eta_{2} & 1+2 \eta_{2}
\end{array}\right) .
\end{aligned}
$$

Again let $\mathbf{w}_{\mathbf{1}}^{\mathbf{n}+\mathbf{1}}=\left(w_{1,0}^{n+1}, \ldots, w_{1_{l, M}}^{n+1}\right)^{t}$ and $\mathbf{w}_{\mathbf{2}}^{\mathbf{n}+\mathbf{1}}=\left(w_{2_{l, 0}}^{n+1}, \ldots, w_{2 l, M}^{n+1}\right)^{t}$, where $(\cdot)^{t}$ represents the transposition of a vector. Along with the homogeneous Neumann boundary conditions, equations (22) and (25) are equivalent to the matrix form

$$
\begin{aligned}
& B_{2} \mathbf{w}_{\mathbf{1}}^{\mathbf{n}+\mathbf{1}}=\mathbf{w}_{\mathbf{1}}^{\mathbf{n}+\frac{2}{3}}, \\
& B_{4} \mathbf{w}_{\mathbf{2}}^{\mathbf{n}+\mathbf{1}}=\mathbf{w}_{\mathbf{2}}^{\mathbf{n}+\frac{2}{3}},
\end{aligned}
$$


where $B_{2}$ and $B_{4}$ are real matrices of size $(M+1) \times(M+1)$

$$
\begin{aligned}
& B_{2}=\left(\begin{array}{cccccccc}
1+2 \eta_{1} & -2 \eta_{1} & 0 & \cdots & \cdots & \cdots & \cdots & 0 \\
-\eta_{1} & 1+2 \eta_{1} & -\eta_{1} & \ddots & & & & \vdots \\
0 & -\eta_{1} & 1+2 \eta_{1} & -\eta_{1} & \ddots & & & \vdots \\
\vdots & \ddots & \ddots & \ddots & \ddots & \ddots & & \vdots \\
\vdots & & \ddots & \ddots & \ddots & \ddots & \ddots & \vdots \\
\vdots & & & \ddots & -\eta_{1} & 1+2 \eta_{1} & -\eta_{1} & 0 \\
\vdots & & & & \ddots & -\eta_{1} & 1+2 \eta_{1} & -\eta_{1} \\
0 & \ldots & \ldots & \ldots & \ldots & 0 & -2 \eta_{1} & 1+2 \eta_{1}
\end{array}\right), \\
& B_{4}=\left(\begin{array}{cccccccc}
1+2 \eta_{2} & -2 \eta_{2} & 0 & \cdots & \cdots & \cdots & \cdots & 0 \\
-\eta_{2} & 1+2 \eta_{2} & -\eta_{2} & \ddots & & & & \vdots \\
0 & -\eta_{2} & 1+2 \eta_{2} & -\eta_{2} & \ddots & & & \vdots \\
\vdots & \ddots & \ddots & \ddots & \ddots & \ddots & & \vdots \\
\vdots & & \ddots & \ddots & \ddots & \ddots & \ddots & \vdots \\
\vdots & & & \ddots & -\eta_{2} & 1+2 \eta_{2} & -\eta_{2} & 0 \\
\vdots & & & & \ddots & -\eta_{2} & 1+2 \eta_{2} & -\eta_{2} \\
0 & \ldots & \ldots & \ldots & \ldots & 0 & -2 \eta_{2} & 1+2 \eta_{2}
\end{array}\right) .
\end{aligned}
$$

Lemma 1 The matrices $B_{i}, i=1,2,3,4$, given in equations (28), (29), (32), and (33) are M-matrices.

Proof The values of $\eta_{1}=d_{w_{1}} \frac{\tau}{h^{2}}>0$ and $\eta_{2}=d_{w_{2}} \frac{\tau}{h^{2}}>0$ because $d_{w_{1}}, d_{w_{2}}, h$, and $\tau$ are positive constants. It follows that the matrices $B_{i}, i=1,2,3,4$, are strictly diagonally dominant. Also entries in the diagonal are positive and off diagonal entries are nonpositive. Therefore, $B_{i}, i=1,2,3,4$, are M-matrices.

Remark 1 The solutions $w_{i}$ appearing in the left-hand side of Eqs. (26), (27), (30), and (31) can be recovered using the inversion of the matrices $B_{i}, i=1,2,3,4$. Since these are M-matrices, by Lemma 1 these, in general, are nonsingular. Therefore these matrices are invertible, and the inverse can be obtained by computational software such as MATLAB.

\subsection{Positivity of the NSFD operator splitting method}

In order to discuss the positivity of the NSFD operator splitting scheme, we use M-matrix theory. A matrix which is real and strictly diagonally dominant is called M-matrix if it has nonpositive off diagonal entries and positive diagonal entries. If a matrix is M-matrix, then it is nonsingular and its inverse matrix has entries of positive numbers [46].

Lemma 2 Equations (20) and (23) guarantee the solutions to be positive under the assumptions of nonnegative initial functions, i.e.,

$$
w_{1 l, m}^{n} \geq 0, \quad w_{2 l, m}^{n} \geq 0 \quad \Longrightarrow \quad w_{1, m}^{n+\frac{1}{3}} \geq 0, \quad w_{2, m}^{n+\frac{1}{3}} \geq 0 .
$$


Remark 2 The verification of a positive solution is demonstrated from equation (20) and (23) as $g_{1}(x, y) \geq 0, g_{2}(x, y) \geq 0$, and all the terms involved in the right-hand sides of (20) and (23) are positive.

Theorem 4 Suppose that the vectors $\mathbf{w}_{1}^{\mathbf{n}+\frac{1}{3}}$ and $\mathbf{w}_{2}^{\mathbf{n}+\frac{1}{3}}$ in matrix representations are positive, then $\mathbf{w}_{1}^{\mathbf{n}+\frac{2}{3}}$ and $\mathbf{w}_{2}^{\mathbf{n}+\frac{2}{3}}$ are likewise positive. If the vectors $\mathbf{w}_{\mathbf{1}}^{\mathbf{n}+\frac{2}{3}}, \mathbf{w}_{\mathbf{2}}^{\mathbf{n}+\frac{2}{3}}$ are positive, then $\mathbf{w}_{1}^{\mathbf{n}+1}$ and $\mathbf{w}_{2}^{\mathbf{n}+\mathbf{1}}$ are likewise positive.

Proof According to Lemma 2, the vectors $\mathbf{w}_{1}^{\mathbf{n}+\frac{1}{3}}$ and $\mathbf{w}_{2}^{\mathbf{n}+\frac{1}{3}}$ are positive. Also Lemma 1 shows that the matrices $B_{1}, B_{2}, B_{3}$, and $B_{4}$ are M-matrices. Therefore entries in the inverse matrices of $B_{1}, B_{2}, B_{3}$, and $B_{4}$ are all positive. It follows that $\mathbf{w}_{1}^{\mathbf{n}+\frac{2}{3}}$ and $\mathbf{w}_{2}^{\mathbf{n}+\frac{2}{3}}$ are positive. Consequently, $\mathbf{w}_{\mathbf{1}}^{\mathbf{n}+\mathbf{1}}$ and $\mathbf{w}_{2}^{\mathbf{n}+\mathbf{1}}$ are also positive.

From the above theorem, it is clear that the proposed method preserves the positivity property, unconditionally.

\subsection{Numerical example}

Simulations of the example are performed by selecting different values of parameters involved in designing the two-dimensional Brusselator chemical reaction model. Different numerical parametric values are chosen for the better illustration and understanding of the claimed discrepancies about the widely used existing numerical scheme and salient features of the proposed numerical scheme. The following example is considered for system (14)-(15) in two dimensions with initial conditions $[47,48]$ :

$$
\begin{array}{ll}
w_{1}(x, y, 0)=0.5+y, & 0 \leq x, y \leq 1, \\
w_{2}(x, y, 0)=1+5 x, & 0 \leq x, y \leq 1,
\end{array}
$$

and homogeneous Neumann boundary conditions.

\subsubsection{Simulations of the forward Euler FD scheme}

In this section we present numerical simulations of Brusselator reaction-diffusion system (14)-(15) with initial conditions (34)-(35) and homogeneous Neumann boundary conditions using the forward Euler FD scheme. The values of parameters $C$ and $D$ are taken as $C=1$ and $D=3.5$ and the values of $d_{w_{1}}$ and $d_{w_{2}}$ are $d_{w_{1}}=d_{w_{2}}=0.002$.

Figure 1 (a) and (b) shows the mesh graph of concentration profile $w_{1}$ and $w_{2}$ at the grid point $(0.9,:)$ along z-direction vs time $t$ using the forward Euler FD scheme. Figure 1 (c) and (d) depicts the two-dimensional plots of profile $w_{1}$ and $w_{2}$ at $x=y=1 \mathrm{vs}$ time $t$. It is clear from Fig. 1 (b) and (d) that $w_{2}$ demonstrates the negative solution of concentration, which is useless.

As system (14)-(15) converges to the equilibrium point $\left(w_{1}^{*}, w_{2}^{*}\right)=(D, C / D)=(3.4$, 0.2941 ) if $1-C+D^{2} \geq 0$ [49], therefore numerical method must converge towards the point of equilibrium of system (14)-(15) at all step sizes of its stable region. All the parts of Fig. 2 tell that the forward Euler numerical scheme cannot find the convergence towards the equilibrium point of Brusselator system (14)-(15) and diverges. 


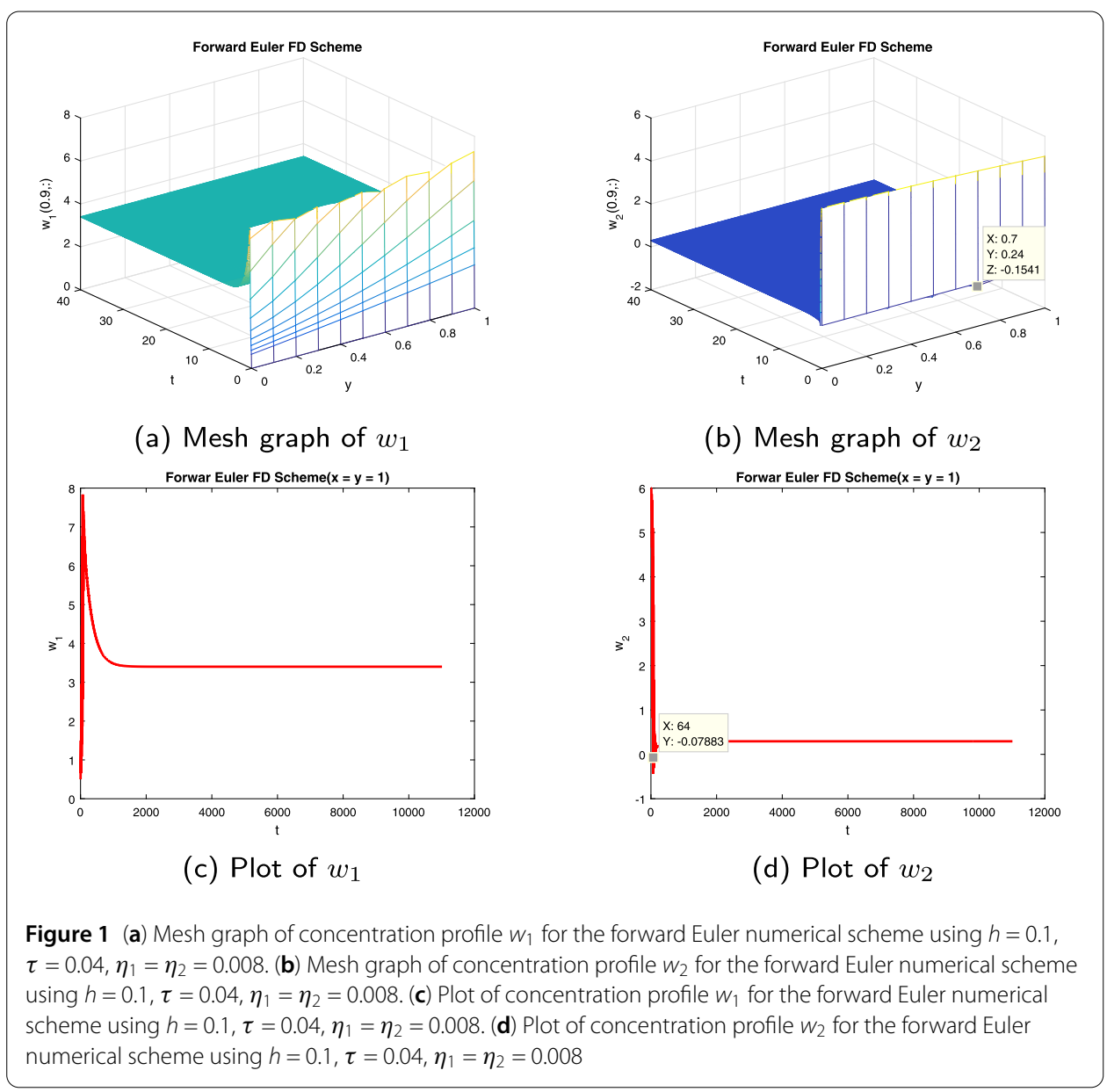

\subsubsection{Simulations of the proposed NSFD operator splitting method}

This section is devoted to the numerical simulation of the Brusselator model with the same values as given in the above section using the proposed NSFD operator splitting method.

Figure 3 (a) and (b) shows the mesh graph of concentration profile $w_{1}$ and $w_{2}$ at the grid point $(0.9$, :) along $\mathrm{z}$-direction vs time $t$ using the proposed NSFD operator splitting method. Figure 3 (c) and (d) depicts the plot of concentration profile $w_{1}$ and $w_{2}$ at $x=$ $y=1 \mathrm{vs}$ time $t$. These graphs verify that the proposed NSFD operator splitting technique preserves the positive solution of the continuous system and also exhibits the convergence towards the point of equilibrium of continuous system (14)-(15).

In Fig. 4, all parts of the figure clearly describe that the proposed NSFD operator splitting method shows the convergence of the system towards the equilibrium point $\left(w_{1}^{*}, w_{2}^{*}\right)$ compared with the forward Euler FD scheme.

In addition, we chose $C=3.4$ and $D=1.0$ and see the behavior of the solution using the proposed NSFD operator splitting method.

It is clearly observed from Figs. 5(a), 5(b) and 6(a), 6(b) that the solutions found using the proposed NSFD operator splitting method are oscillatory and did not converge to the equilibrium point $\left(w_{1}^{*}, w_{2}^{*}\right)$ with the combination of $C=3.4$ and $D=1.0$ so that $1-C+$ $D^{2}<0$. This result agrees with the conclusion made by Twizzel et al. in [49]. 


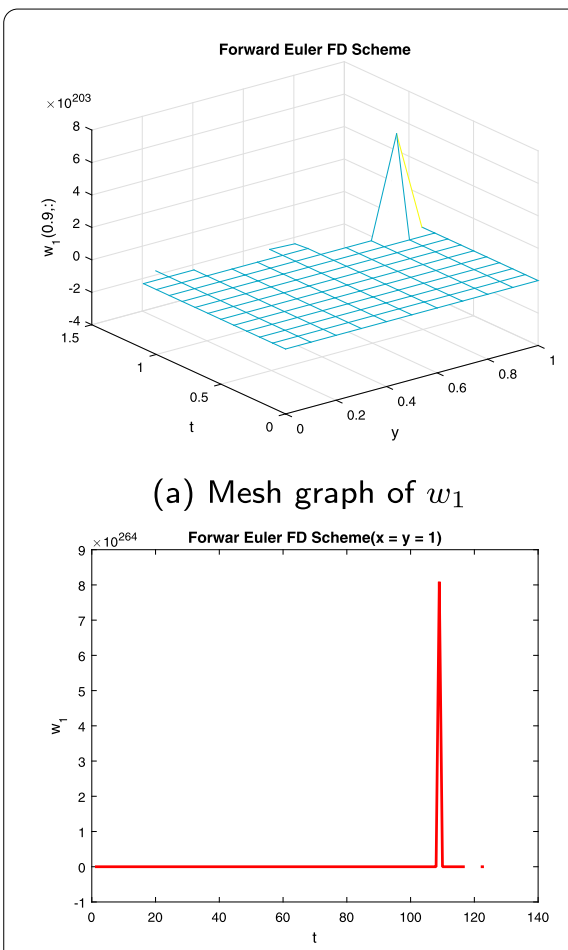

(c) Plot of $w_{1}$

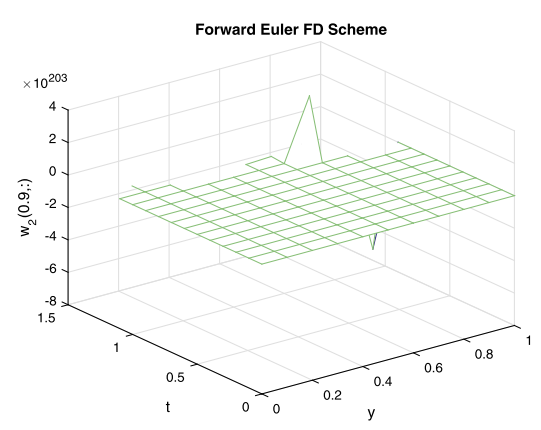

(b) Mesh graph of $w_{2}$

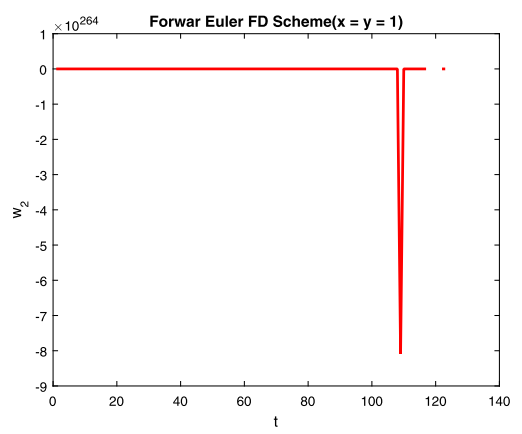

(d) Plot of $w_{2}$

Figure 2 (a) Graph of profile $w_{1}$ for the forward Euler numerical scheme using $h=0.1, \tau=0.1, \eta_{1}=\eta_{2}=0.02$. (b) Graph of profile $w_{2}$ for the forward Euler numerical scheme using $h=0.1, \tau=0.1, \eta_{1}=\eta_{2}=0.02$. (c) Plot of profile $w_{1}$ for the forward Euler numerical scheme using $h=0.1, \tau=0.1, \eta_{1}=\eta_{2}=0.02$. (d) Plot of profile $w_{2}$ for the forward Euler numerical scheme using $h=0.1, \tau=0.1, \eta_{1}=\eta_{2}=0.02$

The comparison of the proposed method and the forward Euler method is presented in Table 1. In this table we show that the exec. (execution) time of NSFD operator splitting technique is better than that of the forward Euler technique.

\section{Glycolysis reaction-diffusion model}

A classic and representative system in the biochemical reaction is glycolysis model. It is a basic biochemical reaction appearing in living cells. The fundamental model was presented by Sel'kov [50] and is represented by two coupled first-order differential equations $[51,52]$. We consider the following two-dimensional glycolysis reaction-diffusion system:

$$
\begin{aligned}
& w_{1_{t}}=d_{w_{1}}\left(w_{1_{x x}}+w_{1_{y y}}\right)+C w_{2}-w_{1}+w_{1}^{2} w_{2}, \\
& w_{2_{t}}=d_{w_{2}}\left(w_{2 x x}+w_{2 y y}\right)+D-C w_{2}-w_{1}^{2} w_{2}
\end{aligned}
$$

with the initial condition

$$
\begin{array}{ll}
w_{1}(x, y, 0)=g_{3}(x, y) \geq 0, & a \leq x, y \leq b, \\
w_{2}(x, y, 0)=g_{4}(x, y) \geq 0, & a \leq x, y \leq b
\end{array}
$$




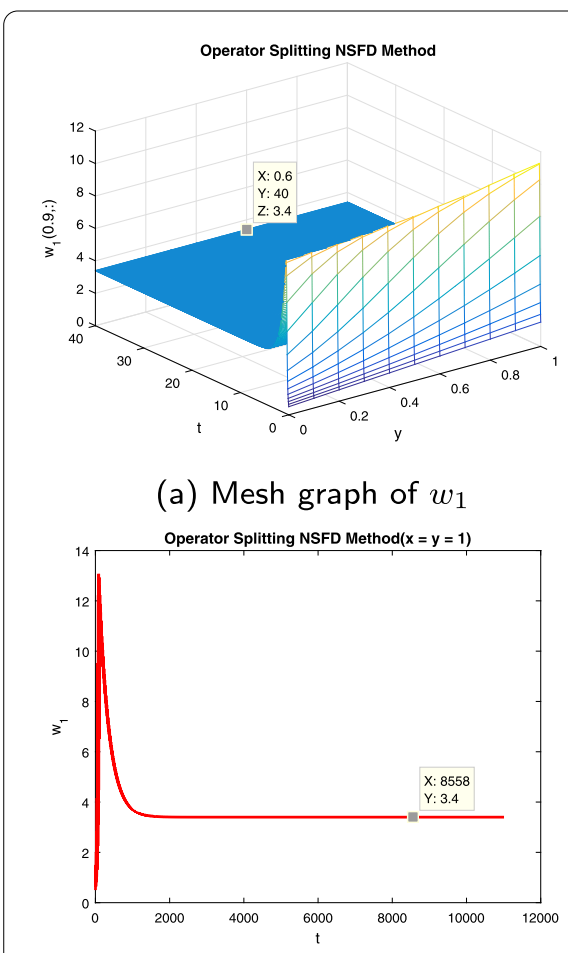

(c) Plot of $w_{1}$

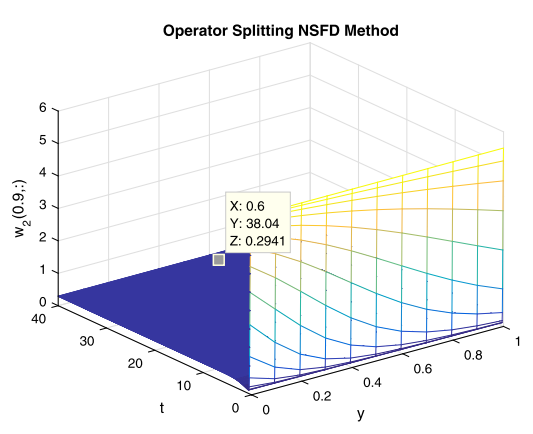

(b) Mesh graph of $w_{2}$

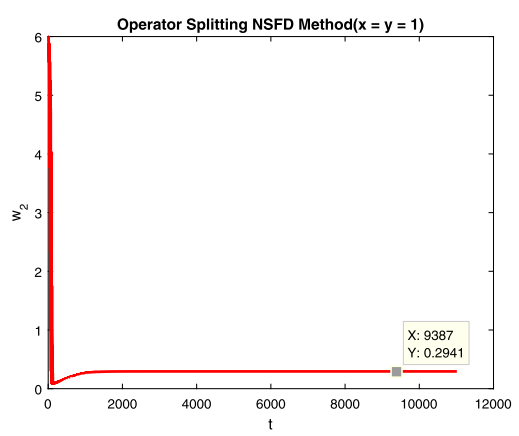

(d) Plot of $w_{2}$

Figure 3 (a) Graph of concentration profile $w_{1}$ using the proposed NSFD splitting method for $h=0.1$, $\tau=0.04, \eta_{1}=\eta_{2}=0.008$. (b) Graph of concentration profile $w_{2}$ using the proposed NSFD splitting method for $h=0.1, \tau=0.04, \eta_{1}=\eta_{2}=0.008$. (c) Plot of concentration $w_{1}$ using the proposed NSFD splitting method for $h=0.1, \tau=0.04, \eta_{1}=\eta_{2}=0.008$. (d) Plot of concentration $w_{2}$ using the proposed NSFD splitting method for $h=0.1, \tau=0.04, \eta_{1}=\eta_{2}=0.008$

and homogeneous Neumann boundary conditions. The equilibrium point of system (36)(37) is $\left(w_{1}^{*}, w_{1}^{*}\right)=\left(D, D /\left(C+D^{2}\right)\right)$. Define

$$
\xi=\frac{D^{4}+(2 C-1) D^{2}+\left(C+C^{2}\right)}{C+D^{2}} .
$$

Strogatz[52] and Mickens[51] discussed that the equilibrium point has the following stability properties:

$$
\begin{aligned}
& \xi>0 \text { : unstable, } \\
& \xi<0 \text { : stable. }
\end{aligned}
$$

Again, after applying the forward Euler FD scheme (10) on system (36)-(37), we have

$$
\begin{aligned}
w_{1_{l, m}}^{n+1}= & w_{1_{l, m}}^{n}+\eta_{1}\left(w_{1_{l-1, m}^{n}}^{n}-2 w_{1_{l, m}}^{n}+w_{1_{l+1, m}}^{n}\right) \\
& +\eta_{1}\left(w_{1_{l, m-1}^{n}}^{n}-2 w_{1_{l, m}}^{n}+w_{1_{l, m+1}}^{n}\right)+\tau C w_{2_{l, m}}^{n}-\tau w_{1_{l, m}}^{n} \\
& +\tau\left(w_{1_{l, m}}^{n}\right)^{2} w_{2_{l, m}}^{n},
\end{aligned}
$$




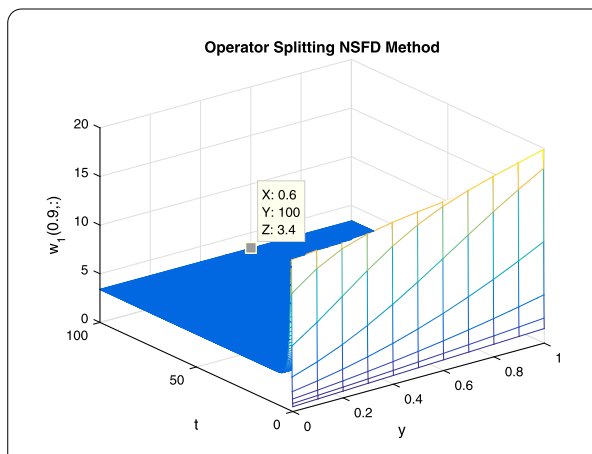

(a) Mesh graph of $w_{1}$

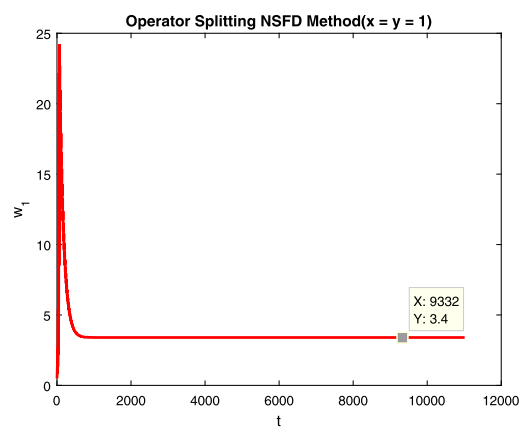

(c) Plot of $w_{1}$

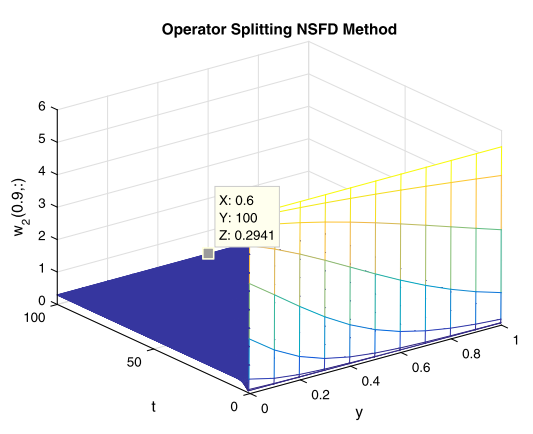

(b) Mesh graph of $w_{2}$

Operator Splitting NSFD Method $(x=y=1)$

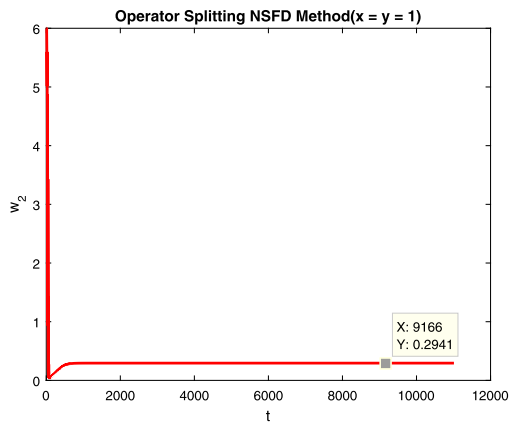

(d) Plot of $w_{2}$

Figure 4 (a) Graph of concentration profile $w_{1}$ by using the proposed NSFD operator splitting method for $h=0.1, \tau=0.1, \eta_{1}=\eta_{2}=0.02$. (b) Graph of concentration $w_{2}$ by using the proposed NSFD operator splitting method for $h=0.1, \tau=0.1, \eta_{1}=\eta_{2}=0.02$. (c) Plot of profile $w_{1}$ by using the proposed NSFD splitting method for $h=0.1, \tau=0.1, \eta_{1}=\eta_{2}=0.02$. (d) Plot of profile $w_{2}$ by using the proposed NSFD splitting method for $h=0.1, \tau=0.1, \eta_{1}=\eta_{2}=0.02$

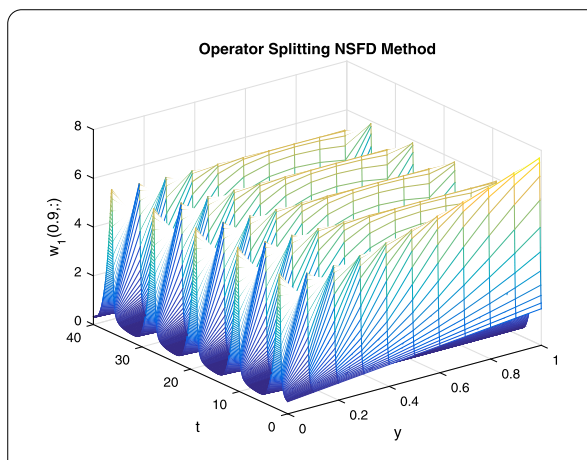

(a) Mesh graph of $w_{1}$

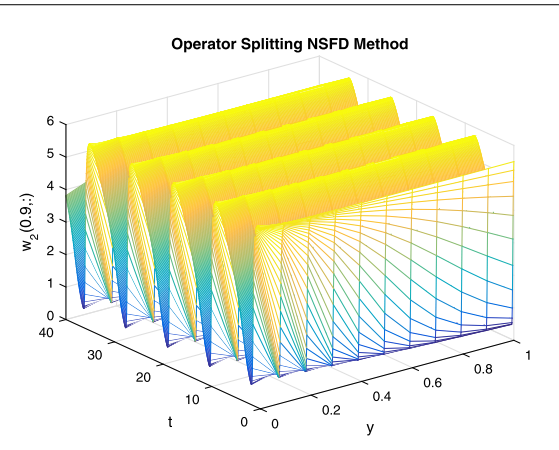

(b) Mesh graph of $w_{2}$

Figure 5 (a) Mesh graph of profile $w_{1}$ by using the proposed NSFD splitting numerical scheme for $h=0.1$, $\tau=0.04, \eta_{1}=\eta_{2}=0.008$. (b) Mesh graph of profile $w_{2}$ by using the proposed NSFD splitting numerical scheme for $h=0.1, \tau=0.04, \eta_{1}=\eta_{2}=0.008$

$$
\begin{aligned}
w_{2_{l, m}}^{n+1}= & w_{2_{l, m}}^{n}+\eta_{2}\left(w_{2_{l-1, m}^{n}}^{n}-2 w_{2_{l, m}}^{n}+w_{2_{l+1, m}}^{n}\right) \\
& +\eta_{2}\left(w_{2_{l, m-1}^{n}}^{n}-2 w_{2_{l, m}^{n}}^{n}+w_{2_{l, m+1}^{n}}^{n}\right)+\tau C w_{1_{l, m}^{n}}^{n} \\
& -\tau\left(w_{1_{l, m}}^{n}\right)^{2} w_{2_{l, m}}^{n} .
\end{aligned}
$$




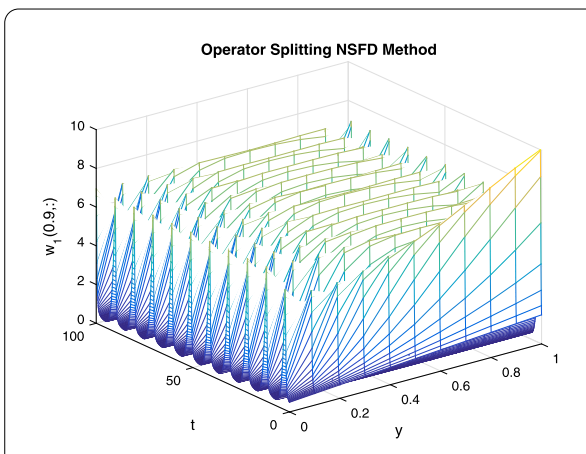

(a) Mesh graph of $w_{1}$

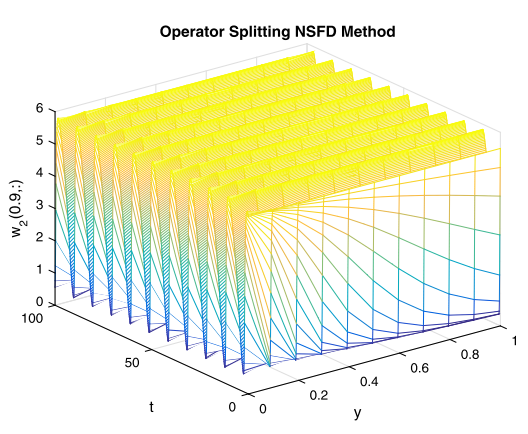

(b) Mesh graph of $w_{2}$

Figure 6 (a) Mesh graph of concentration $w_{1}$ using the proposed NSFD operator numerical scheme for $h=0.1, \tau=0.1, \eta_{1}=\eta_{2}=0.02$. (b) Mesh graph of profile $w_{2}$ using the proposed NSFD splitting numerical scheme for $h=0.1, \tau=0.1, \eta_{1}=\eta_{2}=0.02$

Table 1 Comparison between both numerical methods (Brusselator model)

\begin{tabular}{lllll}
\hline Numerical methods & Step sizes (for exec. time) & Exec. time & Positivity & Convergence \\
\hline Forward Euler & $h=0.1, \tau=0.1$ & 0.4214 Seconds & Failure & Divergence \\
NSFD operator splitting & $h=0.1, \tau=0.1$ & 0.0625 Seconds & Preserved & Convergence \\
\hline
\end{tabular}

The process of the NSFD operator splitting method for equation (36) at the reaction step is

$$
w_{1_{l, m}}^{n+\frac{1}{3}}=\frac{w_{1_{l, m}}^{n}+\tau C w_{2_{l, m}^{n}}^{n}+\tau\left(w_{1_{l, m}^{n}}^{n}\right)^{2} w_{2_{l, m}^{n}}^{n}}{1+\tau}
$$

The NSFD operator splitting method for the space derivative at the second step is

$$
-\eta_{1} w_{1_{l-1, m}}^{n+\frac{2}{3}}+\left(1+2 \eta_{1}\right) w_{1_{l, m}}^{n+\frac{2}{3}}-\eta_{1} w_{1_{l+1, m}}^{n+\frac{2}{3}}=w_{1_{l, m}}^{n+\frac{1}{3}}
$$

Similarly, at the third time step the space derivative w.r.t. $y$ is approximated as

$$
-\eta_{1} w_{1_{l, m-1}}^{n+1}+\left(1+2 \eta_{1}\right) w_{1_{l, m}}^{n+1}-\eta_{1} w_{1_{l, m+1}}^{n+1}=w_{1_{l, m}}^{n+\frac{2}{3}}
$$

and the NSFD method for equation (37)

$$
\begin{aligned}
& w_{2_{l, m}}^{n+\frac{1}{3}}=\frac{w_{2_{l, m}}^{n}+\tau D}{1+\tau C+\tau\left(w_{1_{l, m}}^{n}\right)^{2}}, \\
& -\eta_{2} w_{2_{l-1, m}}^{n+\frac{2}{3}}+\left(1+2 \eta_{2}\right) w_{2_{l, m}^{n+\frac{2}{3}}}^{n-} \eta_{2} w_{2_{l+1, m}}^{n+\frac{2}{3}}=w_{2_{l, m}^{n+\frac{1}{3}}}^{n+} \\
& -\eta_{2} w_{2_{l, m-1}^{n+1}}^{n+1}+\left(1+2 \eta_{2}\right) w_{2_{l, m}^{n+1}}^{n+1}-\eta_{2} w_{2_{l, m+1}^{n+1}}^{n+1}=w_{2_{l, m}}^{n+\frac{2}{3}}
\end{aligned}
$$

The procedure for the verification of unconditional positivity is similar as given in Sects. 4.1 and 4.2. 


\subsection{Example}

In this section, we consider a suitable example of two-dimensional glycolysis model of auto-catalytic chemical reactions. This example elucidates all the attributes and essential features about the proposed scheme and system. The example is given as follows:

$$
\begin{aligned}
& w_{1_{t}}=d_{w_{1}}\left(w_{1_{x x}}+w_{1_{y y}}\right)+C w_{2}-w_{1}+w_{1}^{2} w_{2}, \\
& w_{2_{t}}=d_{w_{2}}\left(w_{2_{x x}}+w_{2_{y y}}\right)+D-C w_{2}-w_{1}^{2} w_{2}
\end{aligned}
$$

and nonnegative initial conditions

$$
\begin{aligned}
& w_{1}(x, y, 0)=1+x+0.5 y, \quad 0 \leq x, y \leq 1, \\
& w_{2}(x, y, 0)=1 / 3, \quad 0 \leq x, y \leq 1,
\end{aligned}
$$

with homogeneous Neumann boundary conditions.

\subsubsection{Simulations of the forward Euler FD scheme}

This section is devoted to the numerical simulations of glycolysis reaction-diffusion system (36)-(37) with initial conditions (51)-(52) and homogeneous Neumann boundary conditions using the forward Euler FD scheme. The values of parameters $C$ and $D$ are taken as $C=3.5$ and $D=0.25$ and the values of $d_{w_{1}}$ and $d_{w_{2}}$ are $d_{w_{1}}=d_{w_{2}}=0.001$.

Figure 7 (a) and (b) depicts the mesh graph of concentration profile $w_{1}$ and $w_{2}$ at the grid point (0.6,:) along z-direction vs time $t$ using the forward Euler FD scheme. Figure 7 (c) and (d) depicts the plot of concentration profile $w_{1}$ and $w_{2}$ at $x=y=1$ vs time $t$. It is clear from Fig. 7 (b) and (d) that the concentration profile $w_{2}$ gives the negative solution of concentration profile, which is meaningless.

As system (36)-(37) converges to the equilibrium point $\left(w_{1}^{*}, w_{2}^{*}\right)=\left(D, D /(C+D)^{2}\right)=$ $(3.4,0.2941)$ if $\xi<0[51,52]$, therefore numerical method must converge to the equilibrium point of glycolysis system (36)-(37) at all step sizes of its stable region. All the parts of Fig. 8 illustrate that the forward Euler numerical scheme fails to find the convergence towards the equilibrium point of glycolysis system (36)-(37) and diverges.

\subsubsection{Simulations of the proposed NSFD operator splitting method}

This section is devoted to the numerical simulation of glycolysis model with the same values as given in the above section using the proposed NSFD operator splitting method.

Figure 9 (a) and (b) shows the mesh graph of the concentration profile $w_{1}$ and $w_{2}$ at the grid point (0.6,:) along z-direction vs time $t$ using the proposed NSFD operator splitting method. Figure 9 (c) and (d) depicts the plot of concentration profile $w_{1}$ and $w_{2}$ at $x=$ $y=1$ vs time $t$. These graphs show that the proposed NSFD splitting scheme preserves the positivity property of the solution of the continuous system and also converges to the equilibrium points of glycolysis continuous system (36)-(37).

In Fig. 10, all parts of the figure clearly describe that the proposed NSFD operator splitting method shows the convergence of system towards the equilibrium point $\left(w_{1}^{*}, w_{2}^{*}\right)$ comparative to the forward Euler FD scheme.

In addition, we chose $C=0.008$ and $D=0.6$ and see the behavior of the solution using the proposed NSFD operator splitting method. 


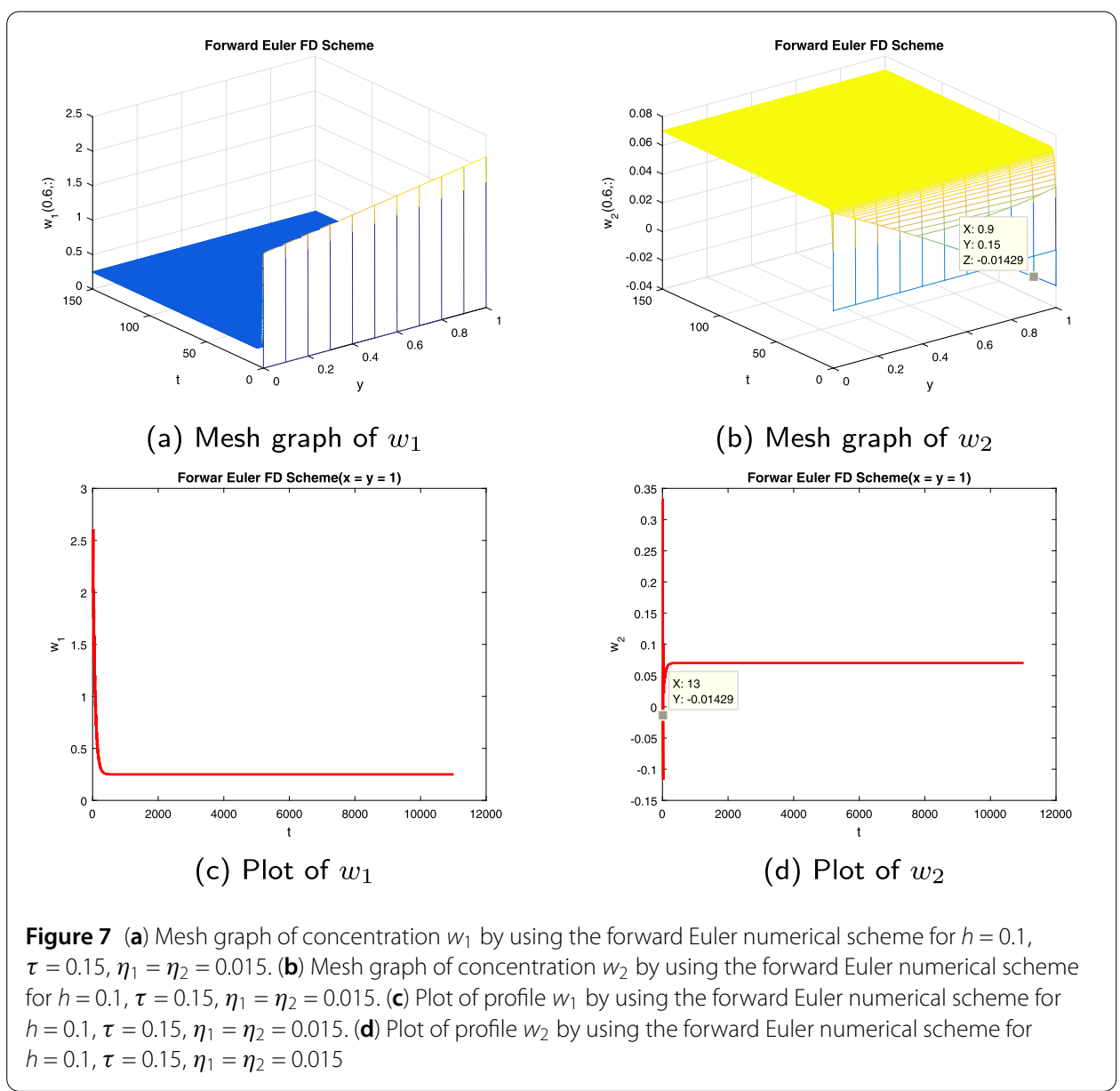

It is clearly observed from Figs. 11(a), 11(b) and 12(a), 12(b) that the solutions found using the proposed NSFD operator splitting method were oscillatory and did not to converge to the equilibrium point $\left(w_{1}^{*}, w_{2}^{*}\right)$ with the combination of $C=0.008$ and $D=0.6$ so that $\xi>0$. Table 2 demonstrates the efficiency of the proposed technique in various aspects for the glycolysis model.

\section{Susceptible-infected-recovered (SIR) epidemic model with saturated incident rate}

In this section, we present an SIR epidemic reaction-diffusion model in two space dimensions, and both techniques are applied on this model. Epidemic models depict the spread of communicable diseases in population through mathematical modeling and give future ideas to control the infection. In these models, an important term is incidence rate which has been considered to ensure that the system of differential equations demonstrates understandable qualitative explanation of the transmission dynamics of diseases [53-56]. A commonly used incidence rate is the bilinear incidence rate defined as the rate at which susceptible population gains infection. These days various modifications of the standard bilinear incidence rate have been used; for instance, saturation incidence rate. In this section, the SIR epidemic model with saturated incidence rate proposed by Suryato [57] is 


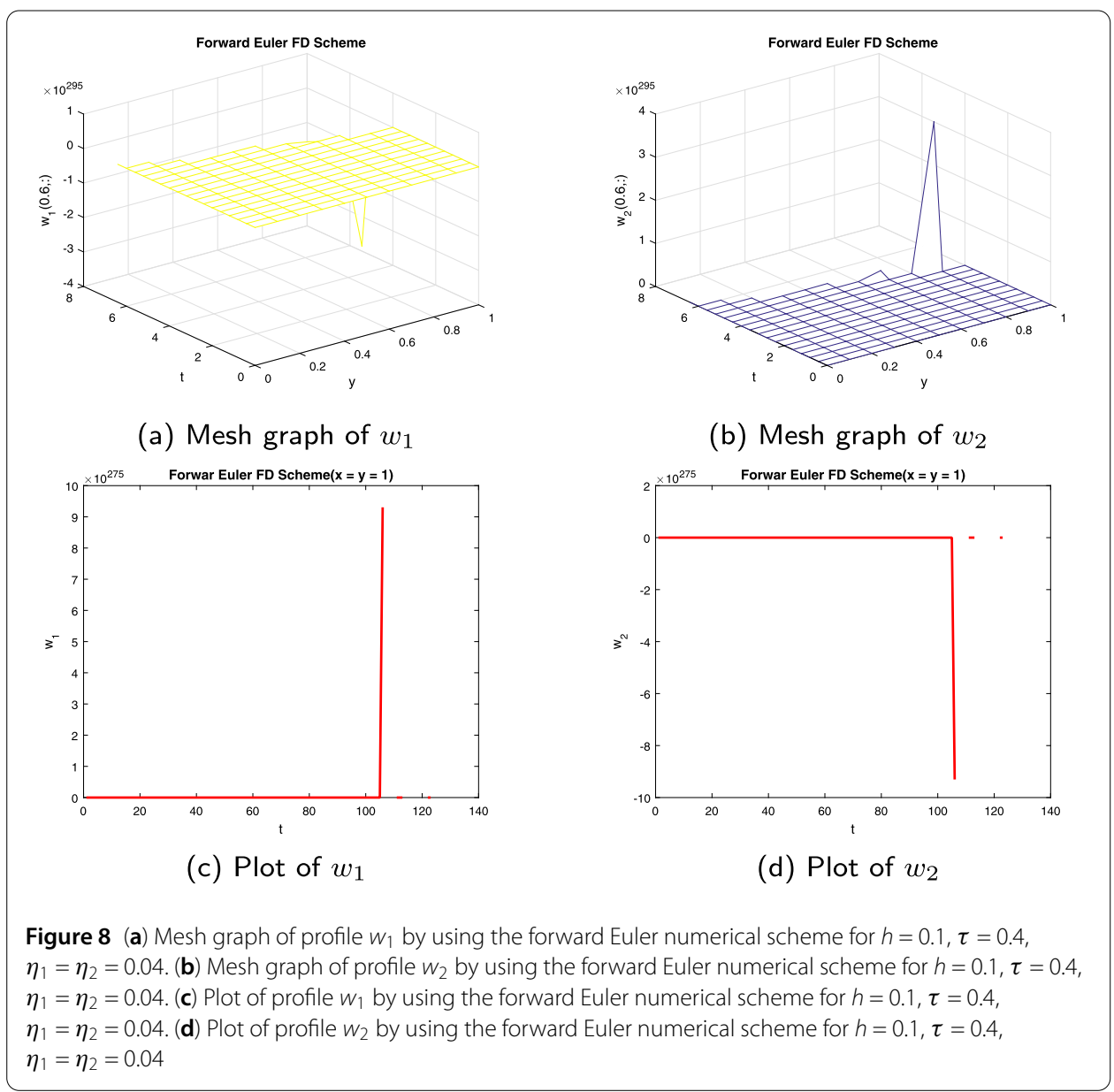

extended to the following reaction-diffusion system:

$$
\begin{aligned}
& w_{1_{t}}=d_{w_{1}}\left(w_{1_{x x}}+w_{1_{y y}}\right)+\mu-\mu w_{1}-\frac{\beta w_{1} w_{2}}{1+\sigma w_{2}}, \\
& w_{2 t}=d_{w_{2}}\left(w_{2_{x x}}+w_{2 y y}\right)+\frac{\beta w_{1} w_{2}}{1+\sigma w_{2}}-(\mu+\gamma) w_{2}, \\
& w_{3 t}=d_{w_{3}}\left(w_{3_{x x}}+w_{3 y y}\right)+\gamma w_{2}-\mu w_{3} .
\end{aligned}
$$

Here, $w_{1}, w_{2}$, and $w_{3}$ represent the susceptible, infected, and recovered individuals. The infection force is measured by $\beta w_{2}$ and the inhibition effect is measured by $\frac{1}{1+\sigma w_{2}}$. The death rate and the birth rate are demonstrated by the parameter $\mu$, the recovery rate from infection is denoted by $\gamma$, and the inhibition parameter is assumed by $\sigma$. All the parameters involved in system (53)-(55) are positive. Since $w_{3}$ is not present in the first two equations, the above system (53)-(55) can be written as

$$
\begin{aligned}
& w_{1_{t}}=d_{w_{1}}\left(w_{1_{x x}}+w_{1_{y y}}\right)+\mu-\mu w_{1}-\frac{\beta w_{1} w_{2}}{1+\sigma w_{2}}, \\
& w_{2_{t}}=d_{w_{2}}\left(w_{2_{x x}}+w_{2 y y}\right)+\frac{\beta w_{1} w_{2}}{1+\sigma w_{2}}-(\mu+\gamma) w_{2}
\end{aligned}
$$




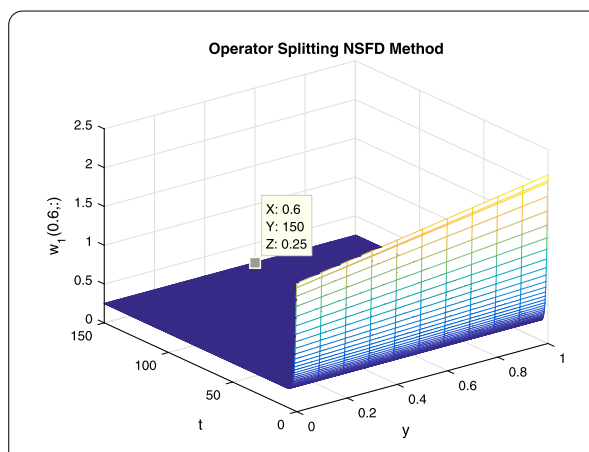

(a) Mesh graph of $w_{1}$

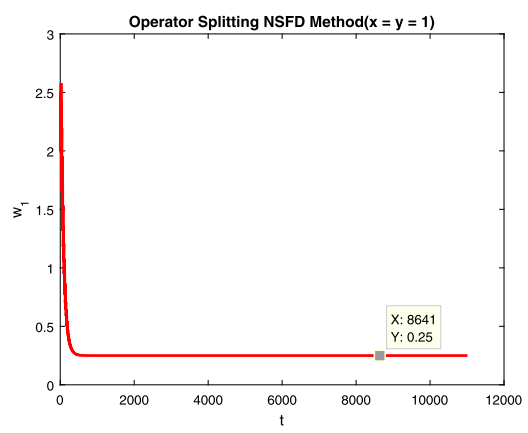

(c) Plot of $w_{1}$

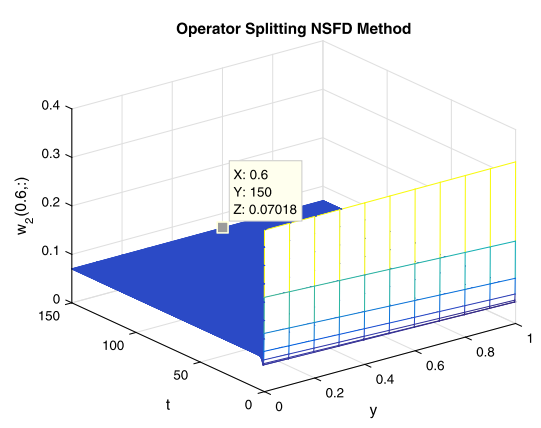

(b) Mesh graph of $w_{2}$

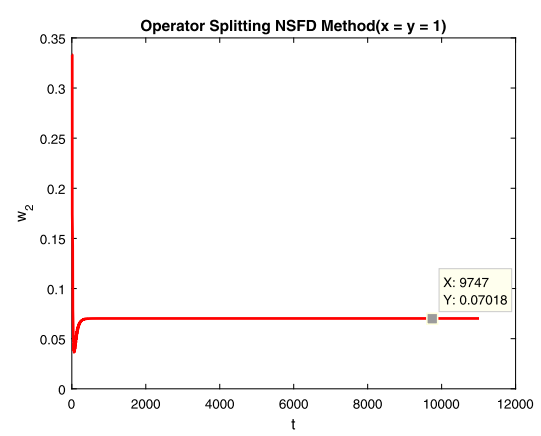

(d) Plot of $w_{2}$

Figure 9 (a) Mesh graph of concentration profile $w_{1}$ by using the proposed NSFD splitting numerical scheme for $h=0.1, \tau=0.15, \eta_{1}=\eta_{2}=0.015$. (b) Mesh graph of profile $w_{2}$ by using the proposed NSFD splitting numerical scheme for $h=0.1, \tau=0.15, \eta_{1}=\eta_{2}=0.015$. (c) Plot of profile $w_{1}$ by using the proposed NSFD splitting numerical scheme for $h=0.1, \tau=0.15, \eta_{1}=\eta_{2}=0.015$. (d) Plot of profile $w_{2}$ by using the proposed NSFD splitting numerical scheme for $h=0.1, \tau=0.15, \eta_{1}=\eta_{2}=0.015$

with the initial condition

$$
\begin{array}{ll}
w_{1}(x, y, 0)=g_{5}(x, y) \geq 0, & a \leq x, y \leq b, \\
w_{2}(x, y, 0)=g_{6}(x, y) \geq 0, & a \leq x, y \leq b
\end{array}
$$

and homogeneous Neumann boundary conditions.

System (56)-(57) possesses two steady states, disease-free steady state (DFSS) and endemic steady state (ESS). DFSS of system $(56)-(57)$ is $(1,0)$ and ESS is $\left(w_{1}^{*}, w_{2}^{*}\right)$, where $w_{1}^{*}=\frac{\mu \sigma+\gamma+\mu}{\mu \sigma+\mathcal{R}_{0}(\gamma+\mu)}$ and $w_{2}^{*}=\frac{\mu\left(\mathcal{R}_{0}-1\right)}{\mu \sigma+\mathcal{R}_{0}(\gamma+\mu)}$. The quantity $\mathcal{R}_{0}$ is the reproductive value that concludes that the disease is erased if $\mathcal{R}_{0}$ is less than one and disease is presented in population if $\mathcal{R}_{0}$ is greater than one.

A similar procedure is adopted for the forward Euler FD scheme (10) on system (56)(57), we get

$$
\begin{aligned}
w_{1_{l, m}}^{n+1}= & w_{1_{l, m}}^{n}+\eta_{1}\left(w_{1_{l-1, m}}^{n}-2 w_{1_{l, m}}^{n}+w_{1_{l+1, m}}^{n}\right) \\
& +\eta_{1}\left(w_{1_{l, m-1}^{n}}^{n}-2 w_{1_{l, m}}^{n}+w_{1_{l, m+1}}^{n}\right)+\tau \mu-\tau \mu w_{1_{l, m}}^{n}-\frac{\tau \beta w_{1} w_{2_{l, m}^{n}}^{n}}{1+\sigma w_{2_{l, m}}^{n}},
\end{aligned}
$$




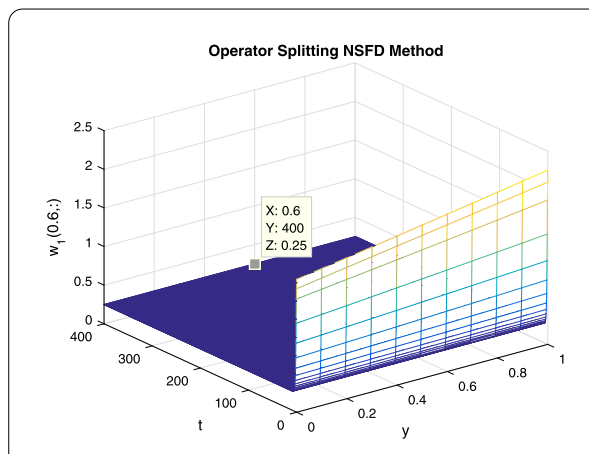

(a) Mesh graph of $w_{1}$

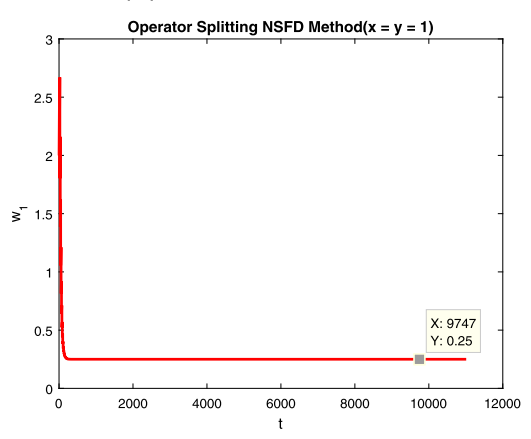

(c) Plot of $w_{1}$

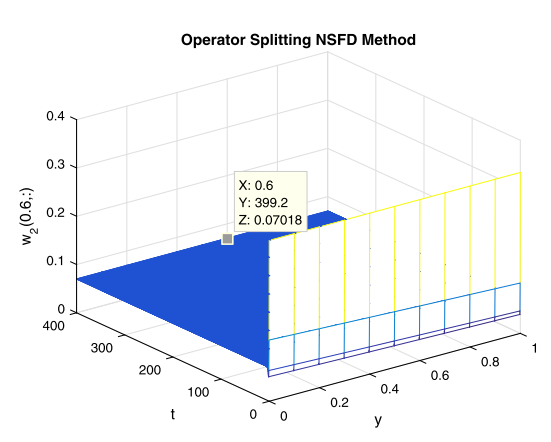

(b) Mesh graph of $w_{2}$

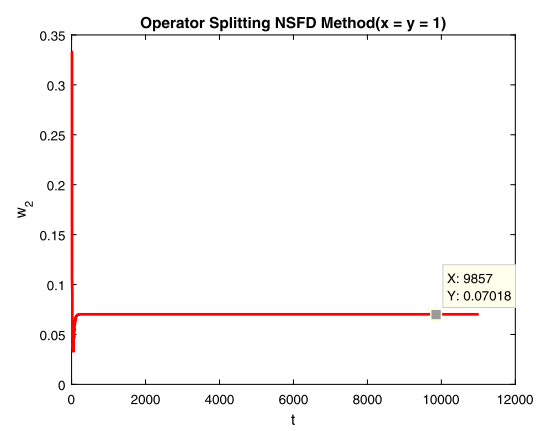

(d) Plot of $w_{2}$

Figure 10 (a) Mesh graph of profile $w_{1}$ by using the proposed NSFD splitting numerical scheme for $h=0.1$, $\tau=0.4, \eta_{1}=\eta_{2}=0.04$. (b) Mesh graph of profile $w_{2}$ by using the proposed NSFD splitting numerical scheme for $h=0.1, \tau=0.4, \eta_{1}=\eta_{2}=0.04$. (c) Plot of profile $w_{1}$ by using the proposed NSFD splitting numerical scheme for $h=0.1, \tau=0.4, \eta_{1}=\eta_{2}=0.04$. (d) Plot of profile $w_{2}$ by using the proposed NSFD splitting method for $h=0.1, \tau=0.4, \eta_{1}=\eta_{2}=0.04$

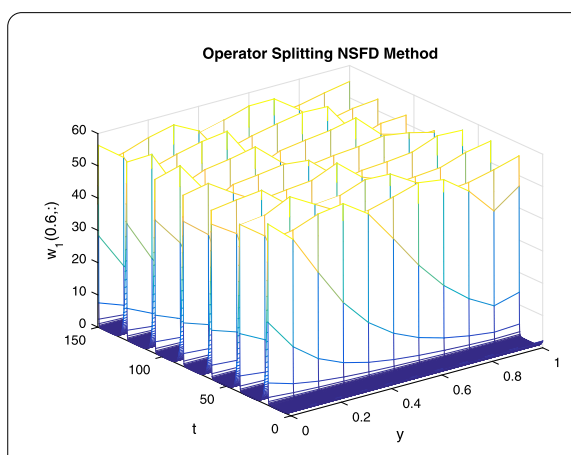

(a) Mesh graph of $w_{1}$

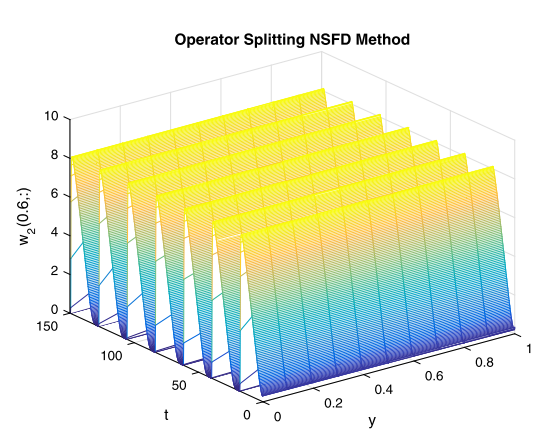

(b) Mesh graph of $w_{2}$

Figure 11 (a) Mesh graph of concentration profile $w_{1}$ by using the proposed NSFD splitting numerical scheme for $h=0.1, \tau=0.04, \eta_{1}=\eta_{2}=0.008$. (b) Mesh graph of profile $w_{2}$ by using the proposed NSFD splitting numerical scheme for $h=0.1, \tau=0.04, \eta_{1}=\eta_{2}=0.008$

$$
\begin{aligned}
w_{2_{l, m}}^{n+1}= & w_{2_{l, m}}^{n}+\eta_{2}\left(w_{2_{l-1, m}^{n}}^{n}-2 w_{2_{l, m}^{n}}^{n}+w_{2_{l+1, m}}^{n}\right) \\
& +\eta_{2}\left(w_{2_{l, m-1}^{n}}^{n}-2 w_{2_{l, m}^{n}}^{n}+w_{2_{l, m+1}}^{n}\right)+\frac{\tau \beta w_{1_{l, m}^{n}}^{n} w_{2_{l, m}}^{n}}{1+\sigma w_{2_{l, m}}^{n}}-\tau(\mu+\gamma) w_{2_{l, m}}^{n} .
\end{aligned}
$$




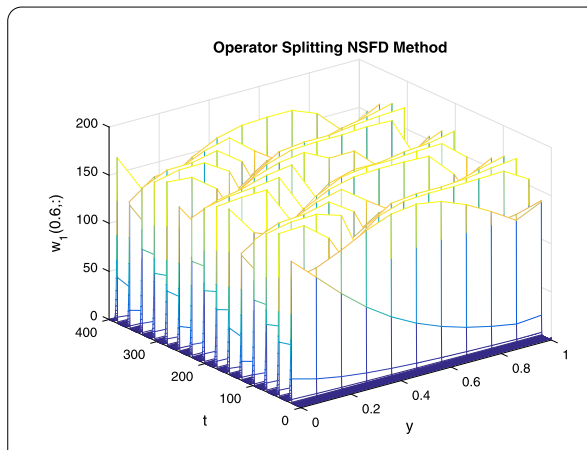

(a) Mesh graph of $w_{1}$

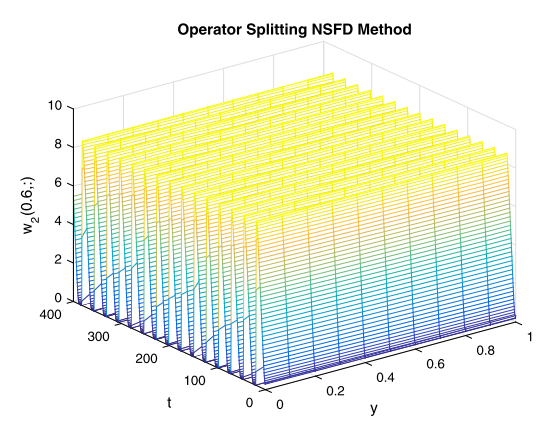

(b) Mesh graph of $w_{2}$

Figure 12 (a) Mesh graph of profile $w_{1}$ by using the proposed NSFD splitting numerical scheme for $h=0.1$, $\tau=0.1, \eta_{1}=\eta_{2}=0.02$. (b) Mesh graph of profile $w_{2}$ by using the proposed NSFD splitting numerical scheme for $h=0.1, \tau=0.1, \eta_{1}=\eta_{2}=0.02$

Table 2 Comparison between both numerical methods (glycolysis model)

\begin{tabular}{lllll}
\hline Numerical methods & Step sizes (for exec. time) & Exec. time & Positivity & Convergence \\
\hline Forward Euler & $h=0.1, \tau=0.4$ & 0.4498 Seconds & Failure & Divergence \\
NSFD operator splitting & $h=0.1, \tau=0.4$ & 0.0639 Seconds & Preserved & Convergence \\
\hline
\end{tabular}

The procedure of the NSFD operator splitting method for equation (56) at reaction step is

$$
w_{1_{l, m}}^{n+\frac{1}{3}}=\frac{w_{1_{l, m}^{n}}^{n}+\tau \mu}{1+\tau \mu+\frac{\tau \beta w_{l, m}^{n}}{1+\sigma w_{2 l, m}^{n}}} .
$$

The NSFD operator splitting method for space derivative at the second step is

$$
-\eta_{1} w_{1_{l-1, m}}^{n+\frac{2}{3}}+\left(1+2 \eta_{1}\right) w_{1_{l, m}^{n+\frac{2}{3}}}^{n}-\eta_{1} w_{1_{l+1, m}}^{n+\frac{2}{3}}=w_{1_{l, m}}^{n+\frac{1}{3}}
$$

Similarly, at the third time step the space derivative w.r.t. $y$ is approximated as

$$
-\eta_{1} w_{1_{l, m-1}}^{n+1}+\left(1+2 \eta_{1}\right) w_{1_{l, m}^{n+1}}^{n+1} \eta_{1} w_{1_{l, m+1}^{n+1}}^{n+1}=w_{1_{l, m}^{n+\frac{2}{3}}}^{n+}
$$

and the NSFD method for equation (57)

$$
\begin{aligned}
& w_{2_{l, m}}^{n+\frac{1}{3}}=\frac{w_{2_{l, m}^{n}}^{n}+\frac{\tau \beta w_{1_{l, m}^{n}}^{n} w_{2_{l, m}^{n}}^{n}}{1+\sigma w_{2 l, m}^{n}}}{1+\tau(\mu+\gamma)}, \\
& -\eta_{2} w_{2_{l-1, m}}^{n+\frac{2}{3}}+\left(1+2 \eta_{2}\right) w_{2_{l, m}^{n+\frac{2}{3}}}^{n}-\eta_{2} w_{2_{l+1, m}}^{n+\frac{2}{3}}=w_{2_{l, m}^{n+\frac{1}{3}}}^{n}, \\
& -\eta_{2} w_{2_{l, m-1}^{n+1}}^{n+1}+\left(1+2 \eta_{2}\right) w_{2_{l, m}^{n+1}}^{n+} \eta_{2} w_{2_{l, m+1}^{n+1}}^{n+1}=w_{2_{l, m}^{n+\frac{2}{3}}}^{n} \text {. }
\end{aligned}
$$

The procedure for the verification of unconditional positivity is similar as given in Sects. 4.1 and 4.2. 


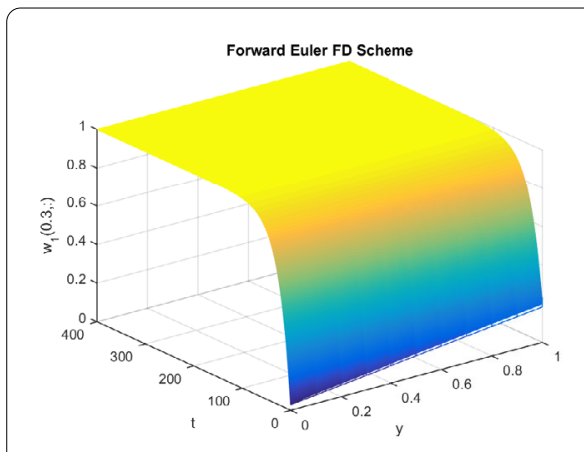

(a) Mesh graph of $w_{1}$

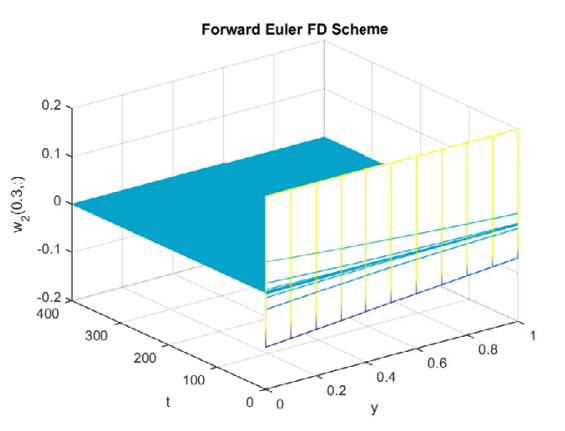

(b) Mesh graph of $w_{2}$

Figure 13 (a) Solution profile of susceptible population $w_{1}$ by using the forward Euler numerical scheme for $h=0.1, \eta_{1}=\eta_{2}=0.0067$. (b) Solution profile of infected population $w_{2}$ by using the proposed NSFD splitting numerical scheme for $h=0.1, \eta_{1}=\eta_{2}=0.0067$

\subsection{Example}

In this section, we consider system (56)-(57) with the following initial conditions:

$$
\begin{aligned}
& w_{1}(x, y, 0)=0.03+0.01 x+0.2 y, \quad 0 \leq x, y \leq 1, \\
& w_{2}(x, y, 0)=0.2, \quad 0 \leq x, y \leq 1
\end{aligned}
$$

The values of the parameters involved in this experiment are $\mu=0.04, \gamma=24, d_{w_{1}}=d_{w_{2}}=$ 0.001 , and $\sigma=1$.

\subsubsection{Simulations of the forward Euler FD scheme}

In this section, we present the behavior of forward Euler method graphically. First we take $\beta=20$ so that the value $\mathcal{R}_{0}=0.83195$, which is less than one, and the system is stable at DFSS.

Figure 13 shows the graphs of forward Euler method at DFSS. In this figure, the graph of infected population describes the negative solution, which is meaningless. For the next figure (Fig. 14), we consider the value $\beta=40$ such that the reproductive value $\mathcal{R}_{0}=1.66389$, which is greater than one. Therefore the system is stable at ESS. But graphs clearly depict that the forward Euler method cannot retain the stability of EES and diverges.

\subsubsection{Simulations of NSFD operator splitting scheme}

This section is devoted to the presentation of simulations for the proposed method. Initially we consider $\beta=20$ so that the value $\mathcal{R}_{0}=0.83195$, which is less than one, and the system is stable at DFSS.

Figure 15 shows the graphs of the proposed splitting method at DFSS. This figure clearly demonstrates that the proposed technique preserves the positive solution of the system under study. For the next figure (Fig. 16), we take the value $\beta=40$ such that the reproductive value $\mathcal{R}_{0}=1.66389$, which is greater than one. Therefore the system is stable at ESS. The NSFD operator splitting method sustains the stability of ESS and converges to the $\operatorname{ESS}\left(w_{1}^{*}, w_{2}^{*}\right)=(0.6013,0.000663)$.

Table 3 also validates the efficacy of the NSFD operator splitting technique in many ways for this model. 


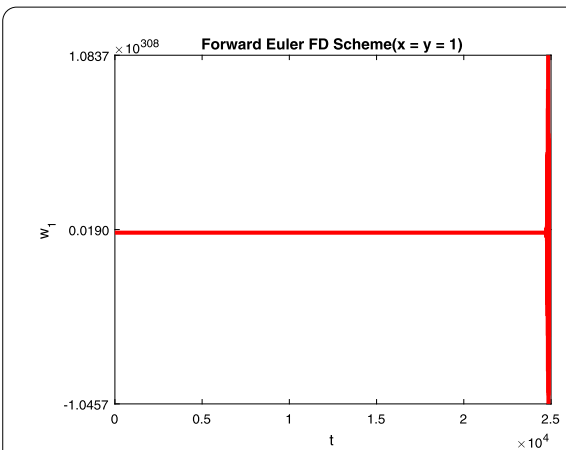

(a) Mesh graph of $w_{1}$

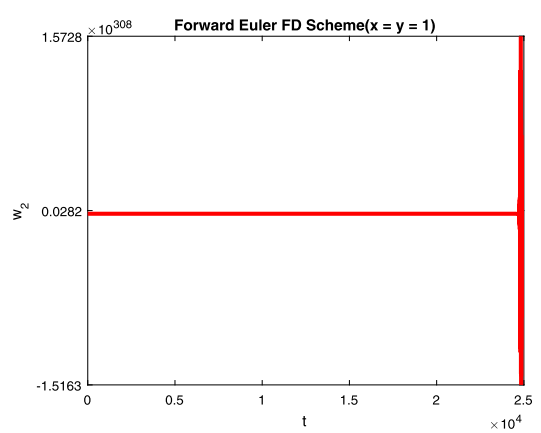

(b) Mesh graph of $w_{2}$

Figure 14 (a) Solution profile of susceptible population $w_{1}$ by using the forward Euler numerical scheme for $h=0.1, \eta_{1}=\eta_{2}=0.0067$. (b) Solution profile of infected population $w_{2}$ by using the proposed NSFD splitting numerical scheme for $h=0.1, \eta_{1}=\eta_{2}=0.0067$

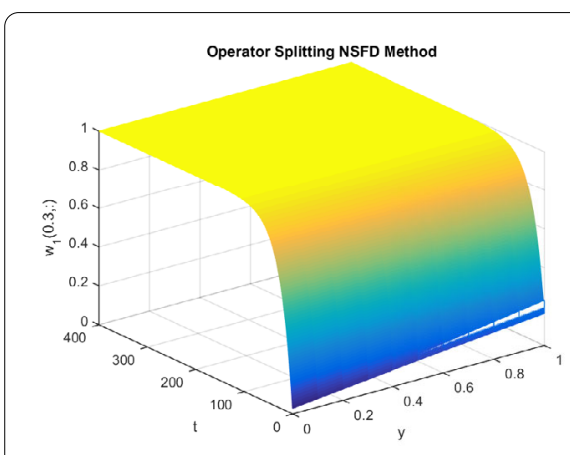

(a) Mesh graph of $w_{1}$

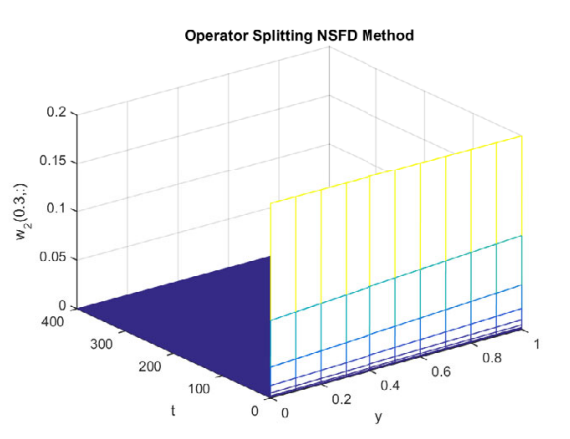

(b) Mesh graph of $w_{2}$

Figure 15 (a) Solution profile of susceptible population $w_{1}$ by using the proposed NSFD splitting numerical scheme for $h=0.1, \eta_{1}=\eta_{2}=0.0067$. (b) Solution profile of infected population $w_{2}$ by using the proposed NSFD splitting numerical scheme for $h=0.1, \eta_{1}=\eta_{2}=0.0067$

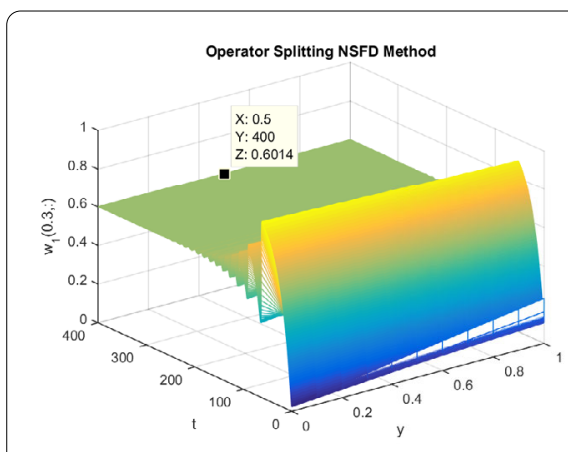

(a) Mesh graph of $w_{1}$

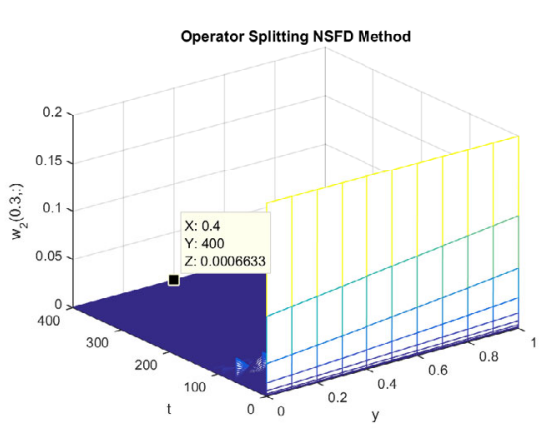

(b) Mesh graph of $w_{2}$

Figure 16 (a) Solution profile of susceptible population $w_{1}$ by using the proposed NSFD splitting numerical scheme for $h=0.1, \eta_{1}=\eta_{2}=0.0067$. (b) Solution profile of infected population $w_{2}$ by using the proposed NSFD splitting numerical scheme for $h=0.1, \eta_{1}=\eta_{2}=0.0067$ 
Table 3 Comparison between both numerical methods (SIR epidemic model)

\begin{tabular}{lllll}
\hline Numerical methods & Step sizes (for exec. time) & Exec. time & Positivity & Convergence \\
\hline Forward Euler & $h=0.1, \tau=0.067$ & 1.9679 Seconds & Failure & Divergence \\
NSFD operator splitting & $h=0.1, \tau=0.067$ & 0.7152 Seconds & Preserved & Convergence \\
\hline
\end{tabular}

\section{Conclusion}

In this work, we design a novel numerical method. This method preserves all the essential conditions demonstrated by the continuous reaction-diffusion systems. The proposed method is the operator splitting NSFD scheme that is unconditionally convergent. The proposed NSFD scheme is implicit in nature but efficient in computation time as compared to the forward Euler FD explicit scheme. For the applications, we take three reaction-diffusion models in two space dimensions and observe that the proposed method shows good agreement with the positivity property and convergence to the stable equilibrium points of reaction-diffusion systems. On the other hand, the forward Euler numerical method fails to retain the property of positivity as well as convergence towards equilibrium points of the given reaction-diffusion system. The figures and tables are presented in this work to verify all the attributes of the proposed NSFD operator splitting method.

\section{Acknowledgements}

The authors wish to thank the anonymous reviewers for their comments and criticism. All of their comments were taken into account in the revised version of the paper, resulting in a substantial improvement with respect to the original submission.

\section{Funding}

No funding.

Availability of data and materials

Not applicable.

\section{Competing interests}

The authors declare that they have no competing interests.

\section{Authors' contributions}

NA, AK, MR, DB, ASA, MAR, and MSI designed the study, developed the methodology, collected the data, performed the analysis, and wrote the manuscript. All authors read and approved the final manuscript.

\section{Author details}

${ }^{1}$ Department of Mathematics, University of Management and Technology, Lahore, Pakistan. ${ }^{2}$ Department of Mathematics, CAKU, Cankiri, Turkey. ${ }^{3}$ Faculty of Engineering, University of Central Punjab, Lahore, Pakistan. ${ }^{4}$ Department of Mathematics, Faculty of Arts and Sciences, Cankaya University, Ankara, Turkey. ${ }^{5}$ Department of Medical Research, China Medical University Hospital, China Medical University, Taichung, Taiwan. ${ }^{6}$ Institute of Space Sciences, Magurele, Bucharest, Romania. ${ }^{7}$ Faculty of Science, Department Mathematics, King Abdulaziz University, Jeddah, Saudi Arabia. ${ }^{8}$ Department Mathematics and Statistics, The University of Lahore, Lahore, Pakistan.

\section{Publisher's Note}

Springer Nature remains neutral with regard to jurisdictional claims in published maps and institutional affiliations.

Received: 6 August 2019 Accepted: 20 April 2020 Published online: 06 May 2020

\section{References}

1. Mickens, R.E.: Nonstandard Finite Difference Models of Differential Equations. World Scientific, Singapore (1994)

2. Fatima, U., Ali, M., Ahmed, N., Rafiq, M.: Numerical modeling of susceptible latent breaking-out quarantine computer virus epidemic dynamics. Heliyon 4, e00631 (2018)

3. Zegeling, P.A., Iqbal, S.: Nonstandard finite differences for a truncated Bratu-Picard model. Appl. Math. Comput. 324 266-284 (2018)

4. Ali, J., Saeed, M., Rafiq, M., Iqbal, S.: Numerical treatment of nonlinear model of virus propagation in computer networks: an innovative evolutionary Pade approximation scheme. Adv. Differ. Equ. 2018, 214 (2018)

5. Obaid, H.A., Ouifki, R., Patidar, K.C.: A nonstandard finite difference method for solving a mathematical model of HIV-TB co-infection. J. Differ. Equ. Appl. 23(2), 1-28 (2017) 
6. Rafiq, M.: Numerical modeling of infectious diseases dynamics. PhD thesis, University of Engineering and Technology, Lahore, Pakistan (2016)

7. Zafar, Z.A., Rehan, K., Mushtaq, M., Rafiq, M.: Numerical treatment for nonlinear Brusselator chemical model. J. Differ. Equ. Appl. 23(3), 521-538 (2016)

8. Ahmed, N., Shahid, N., Iqbal, Z., Jawaz, M., Rafiq, M., Tahira, S.S., Ahmad, M.O.: Numerical modeling of SElQV epidemic model with saturated incidence rate. J. Appl. Environ. Biol. Sci. 8(4) 67-82 (2018)

9. Ahmed, N., Rafiq, M., Rehman, M.A., Iqbal, M.S., Ali, M.: Numerical modelling of three dimensional Brusselator reaction diffusion system. AlP Adv. 9, 015205 (2019)

10. Ahmed, N., Tahira, S.S., Rafiq, M., Rehman, M.A., Ali, M., Ahmad, M.O.: Positivity preserving operator splitting nonstandard finite difference methods for SEIR reaction diffusion model. Open Math. 17, 313-330 (2019)

11. Namjoo, M., Zeinadini, M., Zibaei, S.: Nonstandard finite-difference scheme to approximate the generalized Burgers-Fisher equation. Math. Methods Appl. Sci. 41(17), 8212-8228 (2018)

12. Ahmed, N., Rafiq, M., Rehman, M.A., Ali, M., Ahmad, M.O.: Numerical modeling of SEIR measles dynamics with diffusion. Commun. Math. Appl. 9(3), 315-326 (2018)

13. Ahmed, N., Jawaz, M., Rafiq, M., Rehman, M.A., Ali, M., Ahmad, M.O.: Numerical treatment of an epidemic model with spatial diffusion. J. Appl. Environ. Biol. Sci. 8(6), 17-29 (2018)

14. Chinviriyasit, S., Chinviriyasit, W.: Numerical modeling of SIR epidemic model with diffusion. Appl. Math. Comput. 216 395-409 (2010)

15. Mickens, R.E.: A nonstandard finite difference scheme for a Fisher PDE having nonlinear diffusion. Comput. Math. Appl. 45, 429-436 (2003)

16. Mickens, R.E.: A nonstandard finite difference scheme for an advection-reaction equation. J. Differ. Equ. Appl. 10, 1307-1312 (2004)

17. Al-Showaikh, F., Twizell, E.: One-dimensional measles dynamics. Appl. Math. Comput. 152, 169-194 (2004)

18. Turkyilmazoglu, M.: Accelerating the convergence of decomposition method of Adomian. J. Comput. Sci. 31, 54-59 (2019)

19. Turkyilmazoglu, M.: Convergence accelerating in the homotopy analysis method: a new approach. Adv. Appl. Math. Mech. 10(4), 925-947 (2018)

20. Turkyilmazoglu, M.: Is homotopy perturbation method the traditional Taylor series expansion. Hacet. J. Math. Stat. 44, 651-657 (2015)

21. Turkyilmazoglu, M.: Hyperbolic partial differential equations with nonlocal mixed boundary values and their analytic approximate solutions. Int. J. Comput. Methods 15(1), 1850003 (2018)

22. Turkyilmazoglu, M.: High-order nonlinear Volterra-Fredholm-Hammerstein integro-differential equations and their effective computation. Appl. Math. Comput. 247, 410-416 (2014)

23. Turkyilmazoglu, M.: Parabolic partial differential equations with nonlocal initial and boundary values. Int. J. Comput. Methods 12(5), 121550024 (2015)

24. Korkmaz, A.: Stability satisfied numerical approximates to the non-analytical solutions of the cubic Schrodinger equation. Appl. Math. Comput. 331, 210-231 (2018)

25. Korkmaz, A., Akmaz, H.K.: Numerical simulations of linear transport problem. TWMS J. Appl. Eng. Math. 8(1a), 667-677 (2018)

26. Korkmaz, A.: Simulations of transport in one dimension. Comput. Methods Differ. Equ. 5(3), 189-200 (2017)

27. Korkmaz, A., Ersoy, O., Dag, I:: Motion of patterns modeled by the Gray-Scott autocatalysis system in one dimension. MATCH Commun. Math. Comput. Chem. 77(2), 507-526 (2017)

28. Korkmaz, A., Akmaz, H.K.: Numerical simulations for transport of conservative pollutant. Selçuk J. Appl. Math. 16(1) (2015)

29. Dag, I., Korkmaz, A., Saka, B.: Cosine expansion-based differential quadrature algorithm for numerical solution of the RLW equation. Numer. Methods Partial Differ. Equ. 26(3), 544-560 (2010)

30. Korkmaz, A.: Numerical algorithms for solutions of Korteweg-de Vries equation. Numer. Methods Partial Differ. Equ. 26(6), 1504-1521 (2010)

31. Dag, I., Sahin, A., Korkmaz, A.: Numerical investigation of the solution of Fisher equation via the B-spline Galerkin method. Numer. Methods Partial Differ. Equ. 26, 1483-1503 (2010)

32. Baleanu, D., Jassim, H.K.: A modification fractional homotopy perturbation method for solving Helmholtz and coupled Helmholtz equations on Cantor sets. Fractal Fract. 3, 30 (2019)

33. Baleanu, D., Jassim, H.K.: Approximate solutions of the damped wave equation and dissipative wave equation in fractal strings. Fractal Fract. 3, 26 (2019)

34. Baleanu, D., Shiri, B., Srivastava, H.M., Qurashi, M.A.: A Chebyshev spectral method based on operational matrix for fractional differential equations involving non-singular Mittag-Leffler kernel. Adv. Differ. Equ. 2018, 353 (2018)

35. Ersoy, O., Dag, l.: Numerical solutions of the reaction-diffusion system by using exponential cubic B-spline collocation algorithms. Open Phys. 13, 414-427 (2015)

36. Mittal, R.C., Rohila, R.: Numerical simulation of reaction-diffusion systems by modified cubic B-spline differential quadrature method. Chaos Solitons Fractals 92(1), 1339-1351 (2016)

37. Yanenko, N.N.: The Method of Fractional Steps. Springer, Berlin (1971)

38. Harwood, R.C.: Operator splitting method and applications for semilinear parabolic partial differential equations (Ph.D. dissertation, Pullman; Dept. Math., Washington State Univ (2011)

39. Zharnitsky, V:: Averaging for split-step scheme. Nonlinearity 16, 1359-1366 (2003)

40. Chakrabrty, A., Singh, M., Lucy, B., Ridland, P.: Predator-prey model with pry- taxis and diffusion. Math. Comput. Model. 46, 482-498 (2007)

41. Ansarizadeh, F., Singh, M., Richards, D.: Modelling of tumor cells regression in response to chemotherapeutic treatment. Appl. Math. Model. 48, 96-112 (2017)

42. Naheed, A.: A study of spatio-temporal spread of infectious disease: SARS. Ph.D. thesis, Swinburne University of Technology, Australia (2015)

43. Wang, H.Q.: Numerical studies on the split-step finite difference method for nonlinear Schrodinger equations. Appl. Math. Comput. 170, 17-35 (2005) 
44. Harwood, R.C., Manoranjan, V.S., Edwards, D.B.: Lead-acid battery model under discharge with a fast splitting method. IEEE Trans. Energy Convers. 26(4), 1109-1117 (2011)

45. Prigogine, I., Lefever, R.: Symmetry-breaking instabilities in dissipative systems. J. Chem. Phys. 48, 1695-1700 (1968)

46. Fujimoto, T., Ranade, R.: Two characterizations of inverse-positive matrices: the Hawkins-Simon condition and the Le Chatelier-Braun principle. Electron. J. Linear Algebra 11, 59-65 (2004)

47. Zegeling, P.A., Kok, H.P.: Adaptive moving mesh computations for reaction-diffusion systems. J. Comput. Appl. Math. $168,519-528(2004)$

48. Hu, G., Qiao, Z., Tang, T.: Moving finite element simulations for reaction-diffusion systems. Adv. Appl. Math. Mech. 4(3), 365-381 (2012)

49. Twizell, E.H., Gumel, A.B., Cao, Q.: A second order scheme for the Brusselator reaction-diffusion system. J. Math. Chem. 26, 297-316 (1999)

50. Sel'kov, E.E.: Self-oscillations in glycolysis. A simple model. Eur. J. Biochem. 4, 79-86 (1968)

51. Mickens, R.E.: Positivity preserving discrete model for the coupled ODES modeling glycolysis. In: Proceedings of the Fourth International Conference on Dynamical Systems and Differential Equations, May 24-27 Wilmington, NC, pp. 623-629 (2002)

52. Strogatz, S.H.: Nonlinear Dynamics and Chaos with Applications to Physics, Biology, Chemistry, and Engineering, pp. 205-209. Addison-Wesley, New York (1994)

53. Ahmed, N., Wei, Z., Baleanu, D., Rafiq, M., Rehman, M.A.: Spatio-temporal numerical modeling of reaction-diffusion measles epidemic system. Chaos 29, 103101 (2019)

54. Macias-Diaz, J.E., Ahmed, N., Rafiq, M.: Analysis and nonstandard numerical design of a discrete three-dimensional Hepatitis B epidemic model. Mathematics 7(12), 1157 (2019)

55. Korobeinikov, A., Maini, P.: Nonlinear incidence and stability of infectious disease model. Math. Med. Biol. 22, 113-128 (2005)

56. Xiao, D., Ruan, S.: Global analysis of an epidemic model with nonmonotone incidence rate. Math. Biosci. 208, 419-429 (2007)

57. Suryanto, A.: A dynamically consistent nonstandard numerical scheme for epidemic model with saturated incidence rate. Int. J. Math. Comput. 13, D11 (2011)

\section{Submit your manuscript to a SpringerOpen ${ }^{\circ}$ journal and benefit from:}

- Convenient online submission

- Rigorous peer review

- Open access: articles freely available online

- High visibility within the field

Retaining the copyright to your article

Submit your next manuscript at $\boldsymbol{~ s p r i n g e r o p e n . c o m ~}$ 